\title{
1 Extended cross decomposition for mixed-integer linear programs with strong and weak linking constraints is
}

$4 \quad{ }^{a}$ Department of Chemical Engineering, Queen's University, 19 Division st., Kingston, ON K7L $3 N 6$

\section{Abstract}

Large-scale mixed-integer linear programming (MILP) problems, such as those from twostage stochastic programming, usually have a decomposable structure that can be exploited to design efficient optimization methods. Classical Benders decomposition can solve MILPs with weak linking constraints (which are decomposable when linking variables are fixed) but not strong linking constraints (which are not decomposable even when linking variables are fixed). In this paper, we first propose a new rigorous bilevel decomposition strategy for solving MILPs with strong and weak linking constraints, then extend a recently developed cross decomposition method based on this strategy. We also show how to apply the extended cross decomposition method to two-stage stochastic programming problems with conditional-value-at-risk (CVaR) constraints. In the case studies, we demonstrate the significant computational advantage of the proposed extended cross decomposition method as well as the benefit of including CVaR constraints in stochastic programming.

6 Keywords: Cross decomposition; Benders decomposition; Dantzig-Wolfe

7 decomposition; Risk-averse stochastic programming; Mixed-integer linear

8 programming.

\footnotetext{
(C) 2018. This manuscript version is made available under the CC-BY-NC-ND 4.0 license http://creativecommons.org/licenses/by-nc-nd/4.0/. The final publication is available at elsevier via https://doi.org/10.1016/j.compchemeng.2018.09.011

* Corresponding author

Email address: xiang.li@queensu.ca (Xiang Li)
} 


\section{1. Introduction}

This paper aims at developing an efficient decomposition method to solve large-scale mixed-integer linear programming (MILP) problems in the following form:

$$
\begin{array}{ll}
\min _{x_{0}, x} & c_{0}^{T} x_{0}+c^{T} x \\
\text { s.t. } & B_{1} x_{0}+A_{1} x \leq b_{1}, \\
& B_{2} x_{0}+A_{2} x \leq b_{2}, \\
& x \in X \\
& x_{0} \in X_{0},
\end{array}
$$

10 where variables $x_{0} \in\left\{\left(x_{i}, x_{c}\right): x_{i} \in \mathbb{Z}^{n_{i}}, x_{c} \in \mathbb{R}^{n_{c}}\right\}, x \in \mathbb{R}^{n_{x}}$, parameters $b_{1} \in \mathbb{R}^{m_{1}}$,

${ }_{11} b_{2} \in \mathbb{R}^{m_{2}}$, and other parameters have conformable dimensions. The first two groups of constraints in $(\mathrm{P})$ are called linking constraints in the sense that without them $(\mathrm{P})$ can be decomposed into a number of small problems that are much easier to solve. In other words, linking constraints hinder the decomposition of $(\mathrm{P})$. Among the linking constraints, the 15 second group of constraints are called weak linking constraints in the sense that they do not 16 hinder the decomposition of $(\mathrm{P})$ if $x_{0}$ has fixed values, and the first group of constraints are 17 called strong linking constraints in the sense that they hinder the decomposition of $(\mathrm{P})$ even ${ }_{18}$ if $x_{0}$ has fixed values. Variables in $x_{0}$ are called linking variables. Sets $X$ and $X_{0}$ are defined

19 by linear constraints, and they are assumed to be nonempty and bounded throughout the 20 paper for convenience of discussion.

Many realistic engineering optimization problems can be formulated in form of (P). A typical example is optimization under uncertainty through two-stage stochastic programming, which has been adopted in many areas of process systems engineering, such as supply chain optimization [1], natural gas network design and operation [2], refinery planning [3], expansion of chemical processes [4], etc. In two-stage stochastic programming, the first-stage decisions are to be implemented before the realization of uncertainty, and the second-stage (or recourse) decisions are made to satisfy the second-stage (or recourse) problem for all scenarios addressed by the problem formulation. In the context of two-stage stochastic pro- 
gramming (rigorously speaking, its deterministic equivalent program [5]), Problem (P) can be written as:

$$
\begin{array}{ll}
\min _{x_{0}, x_{1}, \ldots, x_{s}} & \sum_{\omega=1}^{s}\left(c_{0, \omega}^{\mathrm{T}} x_{0}+c_{\omega}^{\mathrm{T}} x_{\omega}\right) \\
\text { s.t. } & B_{1} x_{0}+\sum_{\omega=1}^{s} A_{1, \omega} x_{\omega} \leq b_{1}, \\
& B_{2, \omega} x_{0}+A_{2, \omega} x_{\omega} \leq b_{2, \omega}, \quad \omega \in\{1, \ldots, s\}, \quad(2) \\
& x_{0} \in X_{0}, \\
& x_{\omega} \in X_{\omega}, \quad \omega \in\{1, \ldots, s\},
\end{array}
$$

where $x_{0}$ includes the first-stage variables, $x_{\omega}$ includes the second-stage variables for scenario $\omega$, and there are totally $s$ scenarios addressed. (1) represents strong linking constraints that include $x_{\omega}$ for all scenarios (and therefore hinder the decomposition even when $x_{0}$ is fixed). (2) represents weak linking constraints, each linking first-stage variables and second-stage variables for one scenario. Figure 1 illustrates the structure of Problem (SP). Traditional two-stage stochastic programming formulation (which minimizes an expected cost) includes weak linking constraints but not strong linking constraints. Recently, two variants of twostage stochastic programming formulations have attracted more attention and both of them include strong linking constraints. One variant is risk-averse two-stochastic programming [6] [7] [8] [9], which not only minimizes an expected cost, but also minimizes or limits some risk measure of loss, such as conditional value-at-risk (CVaR) [10]. When the risk measure is bounded in the formulation, the loss in different scenarios need to appear in a same constraint, which is a strong linking constraint. The other variant is chance-constrained two-stage stochastic programming [11] [12] that integrates the notion of chance constrained programming [13] into the two-stage formulation. In this formulation, the first-stage decisions are made such that the probability of the recourse problem being feasible is larger than a predefined level. The probability requirement is modeled with a constraint that includes all scenarios, so this constraint is a strong linking constraint.

When Problem (P) does not include strong linking constraints, it can be efficiently solved by Benders decomposition (BD) [14] (or called L-shaped method [15] when applied 


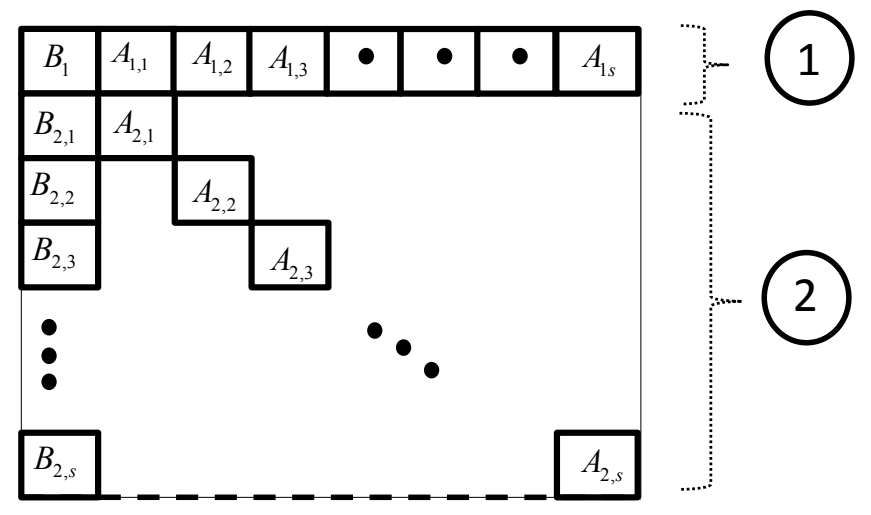

Figure 1: Block structure of constraint (1) and (2) in Problem (SP)

41

to Problem (SP)). BD is efficient because, rather than solving the original problem directly, it solves a sequence of upper bounding problems with fixed $x_{0}$ values and a sequence of lower bounding problems that do not include $x$. Both upper bounding and lower bounding problems are much easier to solve than the original problem. However, when strong linking constraints are present, the upper bounding problems are not decomposable and BD does not have computational advantage. On the one hand, Dantzig-Wolfe decomposition (DWD) [16] [17] or Lagrangian decomposition [18] [19] can be applied to exploit the structure of Problem (P) by dualizing the weak and/or strong linking constraints. However, these methods are rigorous only if Problem $(\mathrm{P})$ has zero dual gap, which is very unlikely considering the problem includes integer variables. While new decomposition methods are needed for solving Problem (P) with both strong linking and weak linking constraints, the relevant research is rarely seen in the literature. The existing ideas for solving Problem $(\mathrm{P})$ include the modification of Benders decomposition method that yields decomposable upper bounding problems [20] [11] and the combination of BD and Lagrangian decomposition [20].

55 The major contribution of this paper is the development of a new decomposition method that is able to efficiently solve Problem $(\mathrm{P})$, based on a novel cross decomposition (CD) method recently developed [21]. The idea of CD was first proposed by Van Roy [22] in 1983; it combines BD with Lagrangian decomposition in order to achieve improved efficiency. Several variants of CD method has then been developed (e.g., [23] [24]), and recently, we 
have developed a novel variant of CD that combines BD and DWD [21]. There are two major advantages of our CD method. One is that the upper bound of the problem can be updated by not only the BD upper bounding problems, but also the DWD upper bounding problems (while CD methods using Lagrangian decomposition update the upper bound only through BD upper bounding problems). The other is that problem infeasibility is handled in a systematic way. However, any CD method (including the one we developed before) requires that the problem can benefit from $\mathrm{BD}$, which is not the case when strong linking constraints are present. Therefore, in this paper, we extend our CD method in order to deal with the strong linking constraints in an efficient way. The key idea is to add another DWD loop in the main CD procedure, so that subproblems coupled by the strong linking constraints can be further decomposed. Furthermore, the additional DWD loop can provide extra cuts and columns to accelerate the main CD procedure.

The remaining part of the paper is structured as follows. In Section 2, we propose a novel bilevel decomposition strategy for solving Problem $(\mathrm{P})$ and prove its validity. The discussion of this strategy motivates the extension of CD. In Section 3, we present extended CD method, which includes a Phase I procedure that prevents infeasible DWD subproblems. In section 4, we discuss in detail how to apply the proposed extended CD method to CVaR constrained two-stage stochastic programming. In section 5, the advantages of CVaR constrained twostage stochastic programming and the proposed solution method are demonstrated via three case study problems adapted from the literature. Relevant conclusions are duly drawn in Section 6.

\section{A bilevel decomposition strategy for $(\mathrm{P})$}

The extended cross decomposition is motivated by a bilevel decomposition strategy. In this strategy, Problem $(\mathrm{P})$ is solved by $\mathrm{BD}$ via viewing $x_{0}$ as complicating variables, and the BD upper bounding problems (or called primal problems), which include strong linking constraints and cannot be directly decomposed, are solved by DWD. Figure 2 illustrate the bilevel decomposition. Here we index the upper level iterations by $k$ and the lower level iterations for each upper level iteration by $l$. 

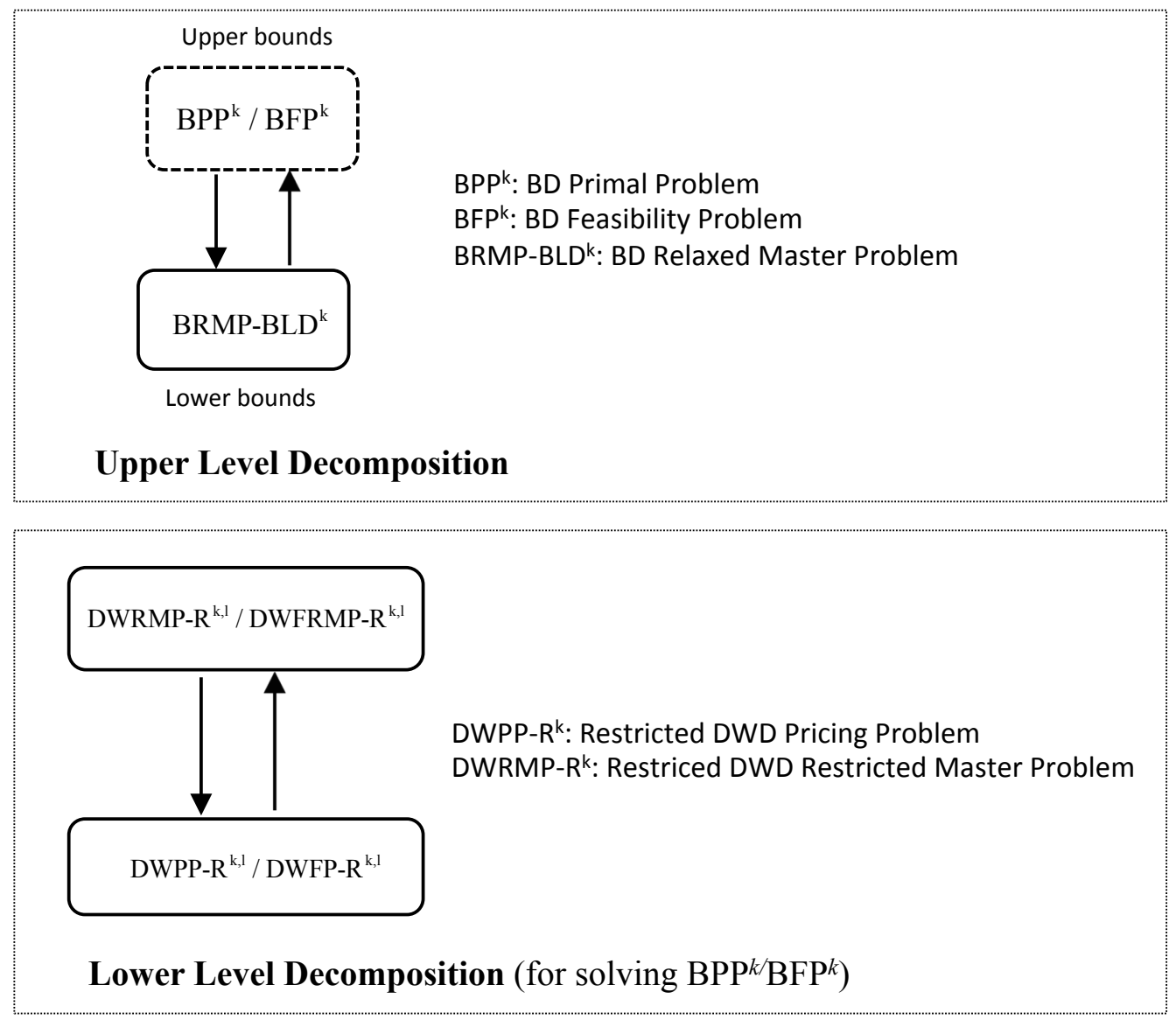

Figure 2: A bilevel decomposition strategy combing BD and DWD

\subsection{The upper level decomposition}

In the upper level, BD is used to solve Problem $(\mathrm{P})$. At each iteration $k, x_{0}$ is fixed to a constant $x_{0}^{k}$, and the following Benders feasibility problem is solved:

$$
\begin{gathered}
o b j_{B F P^{k}}=\min _{x, z_{1} \geq 0, z_{2} \geq 0}\left\|z_{1}\right\|+\left\|z_{2}\right\| \\
\text { s.t. } A_{1} x \leq b_{1}-B_{1} x_{0}^{k}+z_{1}, \\
\quad A_{2} x \leq b_{2}-B_{2} x_{0}^{k}+z_{2}, \\
\quad x \in X
\end{gathered}
$$


where $z_{1}, z_{2}$ are slack variables, and $\|\cdot\|$ denotes any norm function. If $o b j_{B F P}>0$, then Problem $(\mathrm{P})$ is infeasible for $x_{0}=x_{0}^{k}$, and a $\mathrm{BD}$ feasibility cut will be generated. Otherwise, the following Benders primal problem will be solved and a BD optimality cut will be generated:

$$
\begin{gathered}
o b j_{B P P^{k}}=\min _{x} c^{T} x+c_{0}^{T} x_{0}^{k} \\
\text { s.t. } A_{1} x \leq b_{1}-B_{1} x_{0}^{k}, \\
\\
A_{2} x \leq b_{2}-B_{2} x_{0}^{k}, \\
x \in X .
\end{gathered}
$$

Note that $o b j_{B P P^{k}}$ is a valid upper bound for Problem (P). Then the following lower bounding problem (called BD relaxed master problem) is solved at iteration $k$ :

$$
\begin{array}{ll}
\min _{x_{0}, \eta} & \eta \\
\text { s.t. } & \eta \geq o b j_{B P P^{i}}+\left(c_{0}^{T}+\left(\lambda_{1}^{i}\right)^{T} B_{1}\right)\left(x_{0}-x_{0}^{i}\right)+\left(\lambda_{2}^{i}\right)^{T} B_{2}\left(x_{0}-x_{0}^{i}\right), \quad \forall i \in S_{o p t}^{k}, \\
& 0 \geq o b j_{B F P^{i}}+\left(\mu_{1}^{i}\right)^{T} B_{1}\left(x_{0}-x_{0}^{i}\right)+\left(\mu_{2}^{i}\right)^{T} B_{2}\left(x_{0}-x_{0}^{i}\right), \quad \forall i \in S_{\text {feas }}^{k}, \\
& x_{0} \in X_{0} .
\end{array}
$$

$\left(\mathrm{BRMP}-S t d^{k}\right)$

89 Here the first group of constraints are optimality cuts generated in previous iterations $i$ 90 at which Problem $\left(\mathrm{BPP}^{i}\right)$ is feasible, and $\lambda_{1}^{i}, \lambda_{2}^{i}$ are Lagrange multipliers for the strong 91 and weak linking constraints in $\left(\mathrm{BPP}^{i}\right)$, respectively. The second group of constraints are 92 feasibility cuts generated from previous iterations $i$ at which Problem $\left(\mathrm{BPP}^{i}\right)$ is infeasible, ${ }_{93}$ and $\mu_{1}^{i}, \mu_{2}^{i}$ are Lagrange multipliers for the strong and weak linking constraints in $\left(\mathrm{BFP}^{i}\right)$, 94 respectively. Readers are referred to [21] for more discussion on $\left(\mathrm{BRMP}_{9} \mathrm{Std}^{k}\right)$.

95 2.2. The lower level decomposition

Problem $\left(\mathrm{BPP}^{k}\right)$ or $\left(\mathrm{BFP}^{k}\right)$ is not naturally decomposable because of the strong linking constraints, but it can be solved by a DWD procedure for efficient optimization. Specifically, 
$\left(\mathrm{BPP}^{k}\right)$ can be solved by iteratively solving the following two subproblems:

$$
\begin{gathered}
o b j_{D W R M P-R^{k, l}}=\min _{\theta^{0}, \cdots, \theta^{l-1} \geq 0} c^{T}\left(\sum_{i=0}^{l-1} \theta^{i} x^{k, i}\right)+c_{0}^{\mathrm{T}} x_{0}^{k} \\
\text { s.t. } A_{1}\left(\sum_{i=0}^{l-1} \theta^{i} x^{k, i}\right) \leq b_{1}-B_{1} x_{0}^{k}, \\
A_{2}\left(\sum_{i=0}^{l-1} \theta^{i} x^{k, i}\right) \leq b_{2}-B_{2} x_{0}^{k}, \\
\sum_{i=0}^{l-1} \theta^{i}=1 . \\
o b j_{D W P P-R^{k, l}}=\min _{x} c^{\mathrm{T}} x+\left(\lambda_{1}^{k, l}\right)^{\mathrm{T}} A_{1} x \\
\text { s.t. } A_{2} x \leq b_{2}-B_{2} x_{0}^{k}, \\
x \in X .
\end{gathered}
$$

Problem (DWRMP-R ${ }^{k, l}$ ) represents the Dantzig-Wolfe restricted master problem and Problem (DWPP-R $\left.{ }^{k, l}\right)$ represents the Dantzig-Wolfe pricing problem. $l$ indexes the DWD iteration. In (DWRMP-R $\left.\mathrm{R}^{k, l}\right), x^{k, i}$ represents the solution of $\left(\mathrm{DWPP}-\mathrm{R}^{k, i}\right)$ that is obtained in a previous DWD iteration $i(<l)$, and it is often called a column. When $l=1$, an initial column $x^{k, 0}$ is needed to construct (DWRMP- $\mathrm{R}^{k, l}$ ). This initial column can be obtained at the beginning of the algorithm, via solving the following initial pricing problem:

$$
\begin{aligned}
& \min _{x} c^{T} x \\
& \text { s.t. } x \in X .
\end{aligned}
$$

${ }_{96}$ Let the solution of the above problem be $x^{0}$. In the first DWD iteration for solving a $\left(\mathrm{BFP}^{k}\right)$

97 or $\left(\mathrm{BPP}^{k}\right)$, always set $x^{k, 0}=x^{0}$. Lagrange multipliers of the strong linking constraints 98 of (DWRMP- $\mathrm{R}^{k, l}$ ) are represented by $\lambda_{1}^{k, l}$, and they are used to construct (DWPP-R ${ }^{k, l}$ ). $990 b j_{D W R M P-R^{k, l}}$ is a valid upper bound of Problem $\left(\mathrm{BPP}^{k}\right)$, and $o b j_{D W P P-R^{k, l}}+c_{0}^{T} x_{0}^{k}+$ ${ }_{100}\left(\lambda_{1}^{k, l}\right)^{T}\left(B_{1} x_{0}^{k}-b_{1}\right)$ is a valid lower bound of Problem $\left(\mathrm{BPP}^{k}\right)$. The DWD procedure reaches 101 an optimal solution of Problem $\left(\mathrm{BPP}^{k}\right)$ when the upper and lower bounds converge. Readers 
are referred to [21] for more explanation on DWD.

Problem $\left(\mathrm{BFP}^{k}\right)$ can also be solved by a similar DWD procedure. The DWD restricted master problem is:

$$
\begin{gathered}
o b j_{D W F R M P-R^{k, l}}=\min _{\theta^{0}, \cdots, \theta^{l-1}, z_{1}, z_{2} \geq 0}\left\|z_{1}\right\|+\left\|z_{2}\right\| \\
\text { s.t. } A_{1}\left(\sum_{i=0}^{l-1} \theta^{i} x^{k, i}\right) \leq b_{1}-B_{1} x_{0}^{k}+z_{1}, \\
\\
A_{2}\left(\sum_{i=0}^{l-1} \theta^{i} x^{k, i}\right) \leq b_{2}-B_{2} x_{0}^{k}+z_{2}, \\
\sum_{i=0}^{l-1} \theta^{i}=1 .
\end{gathered}
$$

Let $\mu_{1}^{k, l}, \mu_{2}^{k, l}$ be Lagrange multipliers for the strong and weak linking constraints of (DWFRMP-R ${ }^{k, l}$ ), respectively, then the DWD pricing problem can be expressed as:

$$
\begin{gathered}
o b j_{D W F P-R^{k, l}}=\min _{x, z_{2} \geq 0}\left(\mu_{1}^{k, l}\right)^{\mathrm{T}} A_{1} x+\left\|z_{2}\right\| \\
\text { s.t. } A_{2} x \leq b_{2}-B_{2} x_{0}^{k}+z_{2}, \\
\quad x \in X .
\end{gathered}
$$

The proposed DWD procedure for solving Problem $\left(\mathrm{BFP}^{k}\right)$ is similar to but slightly different from the Phase I of a standard DWD procedure, so we prove the validity of the procedure via the next two propositions. The first proposition states that the solution of $\left(\right.$ DWFP-R $\left.{ }^{k, l}\right)$ yields a valid lower bound of Problem $\left(\mathrm{BFP}^{k}\right)$, and the second proposition states the procedure terminates in a finite number of iterations with an optimal solution to Problem $\left(\mathrm{BFP}^{k}\right)$.

Proposition 1. obj $j_{D W F P-R^{k, l}}+\left(\mu_{1}^{k, l}\right)^{T}\left(B_{1} x_{0}^{k}-b_{1}\right)$ is a valid lower bound of Problem $\left(\mathrm{BFP}^{k}\right)$.

Proof. Since (DWFRMP-R ${ }^{k, l}$ ) cannot be unbounded or infeasible, it must have an optimal 
solution. We can express the optimal objective value of $\left(\right.$ DWFRMP-R $\left.{ }^{k, l}\right)$ as:

$$
\begin{aligned}
& o b j_{\text {DWFRMP-R }}, l \\
= & \min _{x \in X^{k, l}, z_{1}, z_{2} \geq 0}\left(\mu_{1}^{k, l}\right)^{T}\left(A_{1} x+B_{1} x_{0}^{k}-b_{1}-z_{1}\right)+\left(\mu_{2}^{k, l}\right)^{T}\left(A_{2} x+B_{2} x_{0}^{k}-b_{2}-z_{2}\right)+\left\|z_{1}\right\|+\left\|z_{2}\right\| \\
= & \left\{\min _{x \in X^{k, l}}\left(\mu_{1}^{k, l}\right)^{T}\left(A_{1} x+B_{1} x_{0}^{k}-b_{1}\right)+\left(\mu_{2}^{k, l}\right)^{T}\left(A_{2} x+B_{2} x_{0}^{k}-b_{2}\right)\right\} \\
& +\left\{\min _{z_{1} \geq 0}\left\|z_{1}\right\|-\left(\mu_{1}^{k, l}\right)^{T} z_{1}\right\}+\left\{\min _{z_{2} \geq 0}\left\|z_{2}\right\|-\left(\mu_{2}^{k, l}\right)^{T} z_{2}\right\},
\end{aligned}
$$

$111 \quad$ where set $X^{k, l}=\left\{x \in \mathbb{R}^{n_{x}}: x=\sum_{i=0}^{l-1} \theta^{i} x^{i}, \sum_{i=1}^{l-1} \theta^{i}=1, \theta^{i} \geq 0, \forall i=0, \cdots, l-1\right\}$.

Suppose $\exists \hat{z}_{1} \geq 0$ such that $\left\|\hat{z}_{1}\right\|-\left(\mu_{1}^{k, l}\right)^{T} \hat{z}_{1} \leq-\epsilon(\epsilon>0)$, then $\forall \alpha>0,\left\|\alpha \hat{z}_{1}\right\|-$ $\left(\mu_{1}^{k, l}\right)^{T} \alpha \hat{z}_{1} \leq-\alpha \epsilon$, which implies that $\min _{z_{1} \geq 0}\left\|z_{1}\right\|-\left(\mu_{1}^{k, l}\right)^{T} z_{1}=-\infty$. This contradicts the fact that $o b j_{\text {DWFRMP-R }}, l$ is finite. Therefore $\left\|z_{1}\right\|-\left(\mu_{1}^{k, l}\right)^{T} z_{1} \geq 0, \forall z_{1} \geq 0$, which results in

$$
\min _{z_{1} \geq 0}\left\|z_{1}\right\|-\left(\mu_{1}^{k, l}\right)^{T} z_{1}=0
$$

(where the minimum value is attained at $z_{1}=0$ ). Similarly,

$$
\min _{z_{2} \geq 0}\left\|z_{2}\right\|-\left(\mu_{2}^{k, l}\right)^{T} z_{2}=0
$$

According to weak duality of Problem $\left(\mathrm{BFP}^{k}\right)$, the following value is a lower bound of $\left(\mathrm{BFP}^{k}\right)$ :

$$
\begin{aligned}
& \min _{\substack{x \in X, z_{1}, z_{2} \geq 0, A_{2} x \leq b_{2}-B_{2} x_{0}^{k}+z_{2}}}\left(\mu_{1}^{k, l}\right)^{T}\left(A_{1} x+B_{1} x_{0}^{k}-b_{1}-z_{1}\right)+\left\|z_{1}\right\|+\left\|z_{2}\right\| \\
= & \left\{\min _{\substack{x \in X, z_{2} \geq 0 \\
A_{2} x \leq b_{2}-B_{2} x_{0}^{k}+z_{2}}}\left(\mu_{1}^{k, l}\right)^{T} A_{1} x+\left\|z_{2}\right\|+\left(\mu_{1}^{k, l}\right)^{T}\left(B_{1} x_{0}^{k}-b_{1}\right)\right\} \\
& +\left\{\min _{z_{1} \geq 0}\left\|z_{1}\right\|-\left(\mu_{1}^{k, l}\right)^{T} z_{1}\right\} . \\
= & o b j_{D W F P-R^{k, l}}+\left(\mu_{1}^{k, l}\right)^{T}\left(B_{1} x_{0}^{k}-b_{1}\right) .
\end{aligned}
$$


Proof. At the DWD iteration $l$, suppose that the solution of $\left(\mathrm{DWFP}^{k, l}\right), x^{k, l}$, is also the solution of a previously solved pricing problem. On the one hand,

$$
o b j_{D W F P-R^{k, l}}=\min _{\substack{z_{2} \geq 0, A_{2} x^{k, l} \leq b_{2}-B_{2} x_{0}^{k}+z_{2}}}\left\|z_{2}\right\|+\left(\mu_{1}^{k, l}\right)^{T} A_{1} x^{k, l} .
$$

On the other hand, the optimal value of (DWFRMP- $\mathrm{R}^{k, l}$ ) can be expressed as:

$$
\begin{aligned}
o b j_{D W F R M P-R^{k, l}}= & \min _{\substack{x \in X^{k, l}, z_{1}, z_{2} \geq 0 \\
A_{2} x \leq b_{2}-B_{2} x_{0}^{k}+z_{2}}}\left\|z_{1}\right\|+\left\|z_{2}\right\|+\left(\mu_{1}^{k, l}\right)^{T}\left(B_{1} x_{0}^{k}+A_{1} x-b_{1}-z_{1}\right) \\
= & \left\{\min _{\substack{x \in X^{k, l}, z_{2} \geq 0 \\
A_{2} x \leq b_{2}-B_{2} x_{0}^{k}+z_{2}}}\left\|z_{2}\right\|+\left(\mu_{1}^{k, l}\right)^{T} A_{1} x\right\}+\left(\mu_{1}^{k, l}\right)^{T}\left(B_{1} x_{0}^{k}-b_{1}\right) \\
& +\left\{\min _{z_{1} \geq 0}\left\|z_{1}\right\|-\left(\mu_{1}^{k, l}\right)^{T} z_{1}\right\} \\
= & \left\{\min _{\substack{x \in X^{k, l}, z_{2} \geq 0 \\
A_{2} x \leq b_{2}-B_{2} x_{0}^{k}+z_{2}}}\left\|z_{2}\right\|+\left(\mu_{1}^{k, l}\right)^{T} A_{1} x\right\}+\left(\mu_{1}^{k, l}\right)^{T}\left(B_{1} x_{0}^{k}-b_{1}\right)
\end{aligned}
$$

where $X^{k, l}=\left\{x \in \mathbb{R}^{n_{x}}: x=\sum_{i=0}^{l-1} \theta^{i} x^{i}, \sum_{i=0}^{l-1} \theta^{i}=1, \theta^{i} \geq 0, \forall i=0, \cdots, l-1\right\}$. Since $x^{k, l}$ has been generated before, it is a point in $X^{k, l}$, so

$$
\min _{\substack{x \in X^{k, l}, z_{2} \geq 0 \\ A_{2} x \leq b_{2}-B_{2} x_{0}^{k}+z_{2}}}\left\|z_{2}\right\|+\left(\mu_{1}^{k, l}\right)^{T} A_{1} x \leq \min _{\substack{z_{2} \geq 0, A_{2} x^{k, l} \leq b_{2}-B_{2} x_{0}^{k}+z_{2}}}\left\|z_{2}\right\|+\left(\mu_{1}^{k, l}\right)^{T} A_{1} x^{k, l}
$$

and therefore

$$
o b j_{D W F R M P-R^{k, l}} \leq o b j_{D W F P-R^{k, l}}+\left(\mu_{1}^{k, l}\right)^{T}\left(B_{1} x_{0}^{k}-b_{1}\right) .
$$


This means that, the upper and lower bounds of Problem $\left(\mathrm{BFP}^{k}\right)$ converge once an extreme point of $X$ is generated at the second time. Since polyhedral set $X$ has only a finite number of extreme points, so the DWD procedure always terminates in a finite number of iterations.

\subsection{Integration of the two levels}

At the termination of the DWD procedure for solving Problem $\left(\mathrm{BPP}^{k}\right)$,

$$
o b j_{B P P^{k}}=o b j_{D W P P-R^{k, l}}+c_{0}^{T} x_{0}^{k}+\left(\lambda_{1}^{k, l}\right)^{T}\left(B_{1} x_{0}^{k}-b_{1}\right)
$$

4

$2 \mathrm{prO}$ denoted by $\left(x_{0}^{*}, x^{*}\right)$.

Let $\lambda_{2}^{k, l}$ be Lagrange multipliers for the weak linking constraints in (DWPP-R ${ }^{k, l}$ ), according to strong duality of (DWPP- $\left.\mathrm{R}^{k, l}\right)$,

$$
o b j_{D W P P-R^{k, l}}=\min _{x \in X} c^{T} x+\left(\lambda_{1}^{k, l}\right)^{T} A_{1} x+\left(\lambda_{2}^{k, l}\right)^{T}\left(A_{2} x-b_{2}+B_{2} x_{0}^{k}\right) .
$$

From equations (1) and (2),

$$
o b j_{B P P^{k}}=\min _{x \in X} c^{T} x+c_{0}^{T} x_{0}^{k}+\left(\lambda_{1}^{k, l}\right)^{T}\left(A_{1} x-b_{1}+B_{1} x_{0}^{k}\right)+\left(\lambda_{2}^{k, l}\right)^{T}\left(A_{2} x-b_{2}+B_{2} x_{0}^{k}\right)
$$

which implies that $\lambda_{1}^{k, l}$ and $\lambda_{2}^{k, l}$ are (optimal) Lagrange multipliers of $\left(\mathrm{BPP}^{k}\right)$. Therefore, the optimality cuts in the standard BD relaxed master problem $\left(\mathrm{BRMP}_{\mathrm{S}} \mathrm{Std}^{k}\right)$ can be written as (via substituting (1)):

$$
\eta \geq o b j_{D W P P-R^{i, j}}+c_{0}^{T} x_{0}+\left(\lambda_{1}^{i, j}\right)^{T}\left(B_{1} x_{0}-b_{1}\right)+\left(\lambda_{2}^{i, j}\right)^{T} B_{2}\left(x_{0}-x_{0}^{i}\right), \quad \forall(i, j) \in T_{o p t}^{k}
$$

where $T_{o p t}^{k}$ includes index pairs that index the BD iteration at which an optimality cut is generated and the last DWD iteration for this BD iteration. We can also rewrite the 
feasibility cuts in a similar way. As a consequence, Problem $\left(\right.$ BRMP-Std $\left.^{k}\right)$ can be rewritten as:

$$
\begin{array}{ll}
\min _{x_{0}, \eta} & \eta \\
\text { s.t. } \quad & \eta \geq o b j_{D W P P-R^{i, j}}+c_{0}^{T} x_{0}+\left(\lambda_{1}^{i, j}\right)^{T}\left(B_{1} x_{0}-b_{1}\right)+\left(\lambda_{2}^{i, j}\right)^{T} B_{2}\left(x_{0}-x_{0}^{i}\right), \\
& \forall(i, j) \in T_{o p t}^{k}, \\
& 0 \geq o b j_{D W F P-R^{i, j}}+\left(\mu_{1}^{i, j}\right)^{T}\left(B_{1} x_{0}-b_{1}\right)+\left(\mu_{2}^{i, j}\right)^{T} B_{2}\left(x_{0}-x_{0}^{i}\right), \quad \forall(i, j) \in T_{\text {feas }}^{k}, \\
& x_{0} \in X_{0} .
\end{array}
$$

$\left(\mathrm{BRMP}-\mathrm{BLD}^{k}\right)$

where $T_{\text {feas }}^{k}$ includes index pairs that index the $\mathrm{BD}$ iteration at which a feasibility cut is generated and the last DWD iteration for this BD iteration. If Problem (BRMP-BLD $\left.{ }^{k}\right)$ is infeasible, then Problem (P) is also infeasible.

Figure 3 provides the flowchart of the bilevel decomposition algorithm. The algorithm considers two tolerances, $\epsilon$ and $\sigma . \epsilon$ is the tolerance for the solution of Problem (P), and it is also the tolerance for the upper level decomposition. $\sigma$ is the tolerance for each lower level DWD procedure. $\epsilon>>\sigma$ is required to ensure the final solution is $\epsilon$-optimal. The DWD loop involving (DWFRMP-R ${ }^{k, l}$ ) and (DWFP-R ${ }^{k, l}$ ) is to solve the feasibility problem $\left(\mathrm{BFP}^{k}\right)$, and the DWD loop involving (DWRMP-R $\left.\mathrm{R}^{k, l}\right)$ and $\left(\mathrm{DWPP}-\mathrm{R}^{k, l}\right)$ is to solve the upper bounding problem $\left(\mathrm{BPP}^{k}\right)$. With Assumption 1, both the upper level BD procedure and the lower level DWD procedures can terminate in a finite number of iterations, so the bilevel decomposition method has the following finite termination property.

Theorem 1. The bilevel decomposition algorithm shown in Figure 3 terminates in finite time with an $\epsilon$-optimal solution or a certification that Problem $(\mathrm{P})$ is infeasible, if Assumption 1 holds and all subproblems can be solved in finite time.

We have not seen in the literature where the proposed bilevel decomposition method was ever developed for solving Problem (P), but there exist methods using similar ideas to deal with strong linking constraints, such as the one developed by by Bruno and Sagastizábal [20]. 


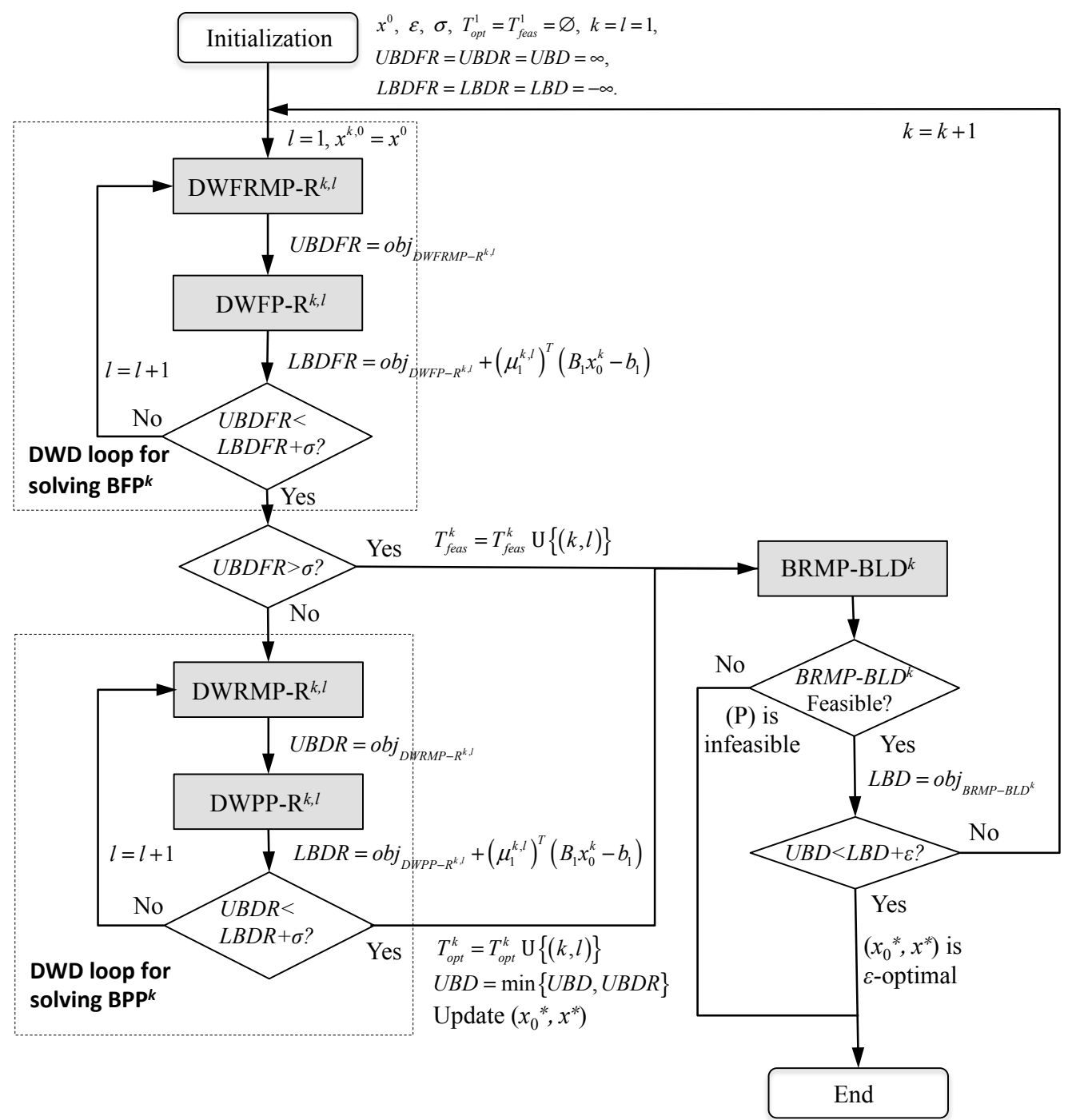

Figure 3: The flowchart of the bilevel decomposition method

In Bruno and Sagastizábal's bilevel decomposition method, the upper level is Lagrangian decomposition and the lower level is $\mathrm{BD}$, but the method is rigorous for $(\mathrm{P})$ only when no integer variables are present, because it requires strong duality of $(\mathrm{P})$. 


\section{The extended cross decomposition method}

\subsection{The basic ECD framework and subproblems}

In this section, we develop a decomposition framework motivated by ideas from bilevel decomposition proposed in section 2 and the cross decomposition method proposed in [21]. In this framework, which is shown in Figure 4, an upper and a lower level decomposition strategy are integrated to efficiently solve Problem $(\mathrm{P})$. At the upper level, a cross decomposition approach for $(\mathrm{P})$ where the following problems; DW restricted master problem, DW pricing problem, Benders primal and feasibility problems $\left(\mathrm{BPP}^{k}\right.$ and $\left.\mathrm{BFP}^{k}\right)$ and Benders relaxed master problems, are iteratively solved is presented. Problems $\left(\mathrm{BPP}^{k}\right)$ and $\left(\mathrm{BFP}^{k}\right)$, that are not decomposable, are then solved via a finitely convergent DWD procedure at the lower level, as in the bilevel decomposition. We refer to this approach as the extended cross decomposition (ECD) approach. The ECD lower level subproblems are same to the lower level subproblems in the bilevel decomposition method proposed in the last section, so we only describe the upper level subproblems here.

At a particular upper level iteration $k$ of ECD, the following DW restricted master problem is solved first:

$$
\begin{aligned}
o b j_{D W R M P-C D^{k}}=\min _{x_{0}, \theta^{i}} & c_{0}^{T} x_{0}+\sum_{i=0}^{k-1} \theta^{i} x^{i} \\
\text { s.t. } & B_{1} x_{0}+A_{1} \sum_{i=0}^{k-1} \theta^{i} x^{i} \leq b_{1}, \\
& B_{2} x_{0}+A_{2} \sum_{i=0}^{k-1} \theta^{i} x^{i} \leq b_{2}, \\
& \sum_{i=0}^{k-1} \theta^{i}=1, \\
& \theta^{i} \geq 0, \\
& x_{0} \in X_{0},
\end{aligned}
$$

where $x^{i}$ is a column generated at the upper level and $i=0,1, \ldots, k-1$ are indices of the generated columns. Problem (DWRMP-CD ${ }^{k}$ ) provides valid upper bounds to Problem (P). 

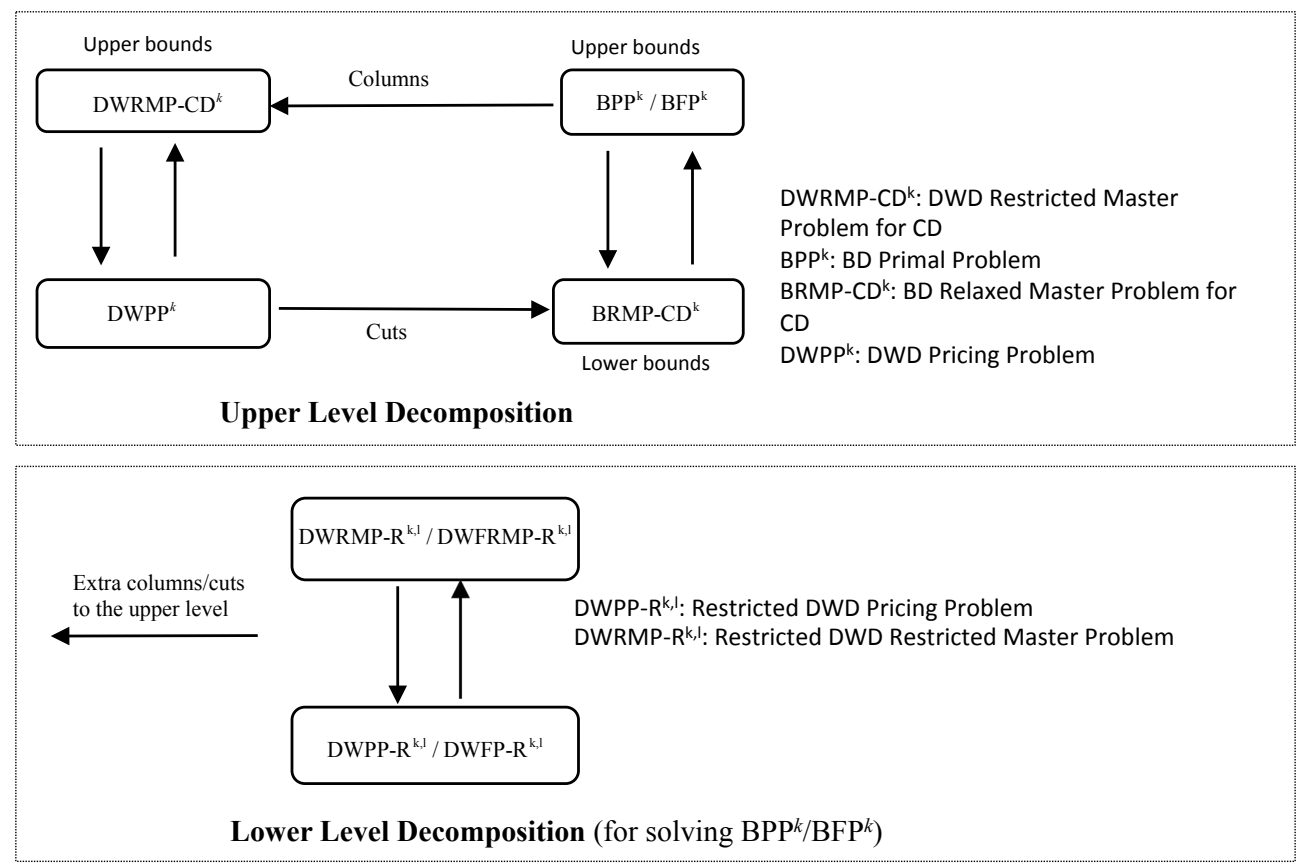

Figure 4: Extended cross decomposition method

Just like in the bilevel decomposition, an initial feasible column, $x^{0} \in X$, is needed to solve Problem (DWRMP-CD ${ }^{k}$ ). It can be obtained by solving the initial pricing problem (IPP). Here we assume that Problem (DWRMP-CD ${ }^{k}$ ) is always feasible for convenience, and later we will discuss how to guarantee the feasibility via a Phase I procedure.

Let $\lambda_{1}^{k}$ and $\lambda_{2}^{k}$ be the Lagrange multiplier associated with strong and weak linking constraints in Problem (DWRMP-CD ${ }^{k}$ ) respectively, then we can construct and solve the following DW pricing problem:

$$
\begin{gathered}
\operatorname{obj}_{D W P P^{k}}=\min _{x} c^{T} x+\left(\lambda_{1}^{k}\right)^{T} A_{1} x+\left(\lambda_{2}^{k}\right)^{T} A_{2} x \\
\text { s.t. } x \in X .
\end{gathered}
$$

69 The solution of Problem $\left(\mathrm{DWPP}^{k}\right)$, denoted by $x^{k}$, provides a new column for Problem $\left(\right.$ DWRMP-CD $\left.{ }^{k}\right)$.

If a full DW iteration is completed and the algorithm is to enter a BD iteration, the 
following Benders relaxed master problem is solved:

$$
\begin{array}{ll}
\min _{x_{0}, \eta} \eta & \\
\text { s.t. } & \eta \geq o b j_{D W P P^{i}}+c_{0}^{T} x_{0}+\left(\lambda_{1}^{i}\right)^{\mathrm{T}}\left(B_{1} x_{0}-b_{1}\right)+\left(\lambda_{2}^{i}\right)^{\mathrm{T}}\left(B_{2} x_{0}-b_{2}\right), \quad \forall i \in U_{o p t}^{k}, \\
& \eta \geq o b j_{D W P P-R^{i, j}}+c_{0}^{T} x_{0}+\left(\lambda_{1}^{i, j}\right)^{\mathrm{T}}\left(B_{1} x_{0}-b_{1}\right)+\left(\lambda_{2}^{i, j}\right)^{\mathrm{T}} B_{2}\left(x_{0}-x_{0}^{i}\right), \quad \forall(i, j) \in T_{o p t}^{k}, \\
& 0 \geq o b j_{D W F P-R^{i, j}}+\left(\mu_{1}^{i, j}\right)^{\mathrm{T}}\left(B_{1} x_{0}-b_{1}\right)+\left(\mu_{2}^{i, j}\right)^{\mathrm{T}} B_{2}\left(x_{0}-x_{0}^{i}\right), \quad \forall(i, j) \in T_{\text {feas }}^{k}, \\
& x_{0} \in X_{0},
\end{array}
$$

$\left(\mathrm{BRMP}_{-} \mathrm{CD}{ }^{k}\right)$

where $U_{o p t}^{k}$ includes the indices of all previous iterations in which Problem $\left(\mathrm{DWPP}^{k}\right)$ is solved, while $T_{\text {opt }}^{k}$ and $T_{\text {feas }}^{k}$ have been defined in Section 2. The optimality and feasibility cuts in $\left(\mathrm{BRMP}-\mathrm{CD}^{k}\right)$ come from previous BD iterations and DW iterations, and readers are referred to [21] for more discussions on $\left(\mathrm{BRMP}-\mathrm{CD}^{k}\right)$.

Note that in ECD, DW restricted master problems and DW pricing problems are included in both the upper level and the lower level. However, in the upper level, both strong and weak linking constraints complicate the problem and therefore they are dualized in the DW pricing problems, while in the lower level, only strong linking constraints complicate the problem (because the weak linking constraints no longer complicate the problem when $x_{0}$ is fixed) and therefore weak linking constraints are not dualized in the DW pricing problem.

\subsection{Synergizing the upper and the lower level}

The two levels in ECD can be synergized to yield stronger bounds and accelerate convergence, like the synergy of DWD and BD iterations within the CD method. Specifically, columns and cuts can be generated from the solutions of the DW pricing problems at the lower level, and they can be added to Problem $\left(\right.$ DWRMP-CD $\left.{ }^{k}\right)$ and $\left(\mathrm{BRMP}^{-C D}{ }^{k}\right)$ at the upper level in order to yield better upper and lower bounds for the original problem $(\mathrm{P})$. 
As a result, the DW restricted master problem can be enhanced as:

$$
\begin{array}{ll}
\min _{x_{0}, \theta^{i}} & c_{0}^{T} x_{0}+\sum_{i=0}^{t-1} \theta^{i} \hat{x}^{i} \\
\text { s.t. } & B_{1} x_{0}+A_{1} \sum_{i=0}^{t-1} \theta^{i} \hat{x}^{i} \leq b_{1}, \\
& B_{2} x_{0}+A_{2} \sum_{i=0}^{t-1} \theta^{i} \hat{x}^{i} \leq b_{2}, \\
& \sum_{i=0}^{t-1} \theta^{i}=1, \\
& \theta^{i} \geq 0, \\
& x_{0} \in X_{0},
\end{array}
$$

where $\hat{x}^{i}$ represent a column generated from either the upper level or the lower level, and $t$ indexes all the columns. If $\hat{x}^{i}$ is generated at the $k$ th upper level iteration, then $\hat{x}^{i}=x^{k}$. If $\hat{x}^{i}$ is generated at the $l$ th lower level iteration for the $k$ th upper level iteration, then $\hat{x}^{i}=x^{k, l}$.

The enhanced Benders relaxed master problem can be written as:

$$
\begin{array}{ll}
\min _{x_{0}, \eta} \eta & \\
\text { s.t. } & \eta \geq o b j_{D W P P^{i}}+c_{0}^{T} x_{0}+\left(\lambda_{1}^{i}\right)^{\mathrm{T}}\left(B_{1} x_{0}-b_{1}\right)+\left(\lambda_{2}^{i}\right)^{\mathrm{T}}\left(B_{2} x_{0}-b_{2}\right), \quad \forall i \in U_{o p t}^{k}, \\
& \eta \geq o b j_{D W P P-R^{i, j}}+c_{0}^{T} x_{0}+\left(\lambda_{1}^{i, j}\right)^{\mathrm{T}}\left(B_{1} x_{0}-b_{1}\right)+\left(\lambda_{2}^{i, j}\right)^{\mathrm{T}} B_{2}\left(x_{0}-x_{0}^{i}\right), \quad \forall(i, j) \in \hat{T}_{o p t}^{k}, \\
& 0 \geq o b j_{D W F P-R^{i, j}}+\left(\mu_{1}^{i, j}\right)^{\mathrm{T}}\left(B_{1} x_{0}-b_{1}\right)+\left(\mu_{2}^{i, j}\right)^{\mathrm{T}} B_{2}\left(x_{0}-x_{0}^{i}\right), \quad \forall(i, j) \in \hat{T}_{f e a s}^{k}, \\
& x_{0} \in X_{0},
\end{array}
$$

186 where $\hat{T}_{\text {opt }}^{k}$ includes index pairs that index the BD iteration at which an optimality cut is generated and all DWD iterations for this BD iteration, while $\hat{T}_{\text {feas }}^{k}$ includes index pairs that index the BD iteration at which a feasibility cut is generated and all DWD iterations for this BD iteration. 
Figure 5 shows the algorithmic flowchart of ECD. The left part of the flowchart depicts the ECD upper level, where Problems (DWRMP-ECD $\left.{ }^{k}\right),\left(\mathrm{DWPP}^{k}\right)$ and $\left(\mathrm{BRMP}^{\mathrm{E}} \mathrm{ECD}^{k}\right)$ are solved, and the right part of the flowchart depicts the ECD lower level, where Problems $\left(\right.$ DWFRMP-R $\mathrm{R}^{k, l}$ ) and (DWFP-R $\mathrm{R}^{k, l}$ ) are solved iteratively. The finite convergence property of the lower level procedure is proved in the last section, and the finite convergence property of the upper level CD procedures is proved in [21], so we have the following finite convergence property of ECD.

Theorem 2. If Assumption 1 holds and all subproblems can be solved in finite time, then the extended cross decomposition method in Figure 5 terminates in a finite number of steps with an $\epsilon$-optimal solution of Problem $(\mathrm{P})$ or a certification that Problem $(\mathrm{P})$ is infeasible.

\subsection{Further discussions}

\subsubsection{Phase 1 procedure and subproblems}

In this section, we present a Phase I procedure to prevent the infeasibility of Problem (DWRMP-ECD ${ }^{k}$ ). The Phase I procedure essentially solves the following feasibility problem:

$$
\begin{gathered}
o b j_{F P}=\min _{x_{0}, x, z_{1} \geq 0, z_{2} \geq 0}\left\|z_{1}\right\|+\left\|z_{2}\right\| \\
\text { s.t. } A_{1} x \leq b_{1}-B_{1} x_{0}+z_{1}, \\
\\
A_{2} x \leq b_{2}-B_{2} x_{0}+z_{2}, \\
\quad x \in X, \\
\quad x_{0} \in X_{0},
\end{gathered}
$$

where $z_{1}$ and $z_{2}$ are slack variables. Problem (FP) is always feasible with the assumption that $X$ and $X_{0}$ are nonempty. The Phase I procedure actually employs ECD to solve Problem (FP), and the subproblems need to be solved in this procedure is illustrated in Figure 6. At iteration $k$ at the upper level, the following DW restricted master problem is solved: 


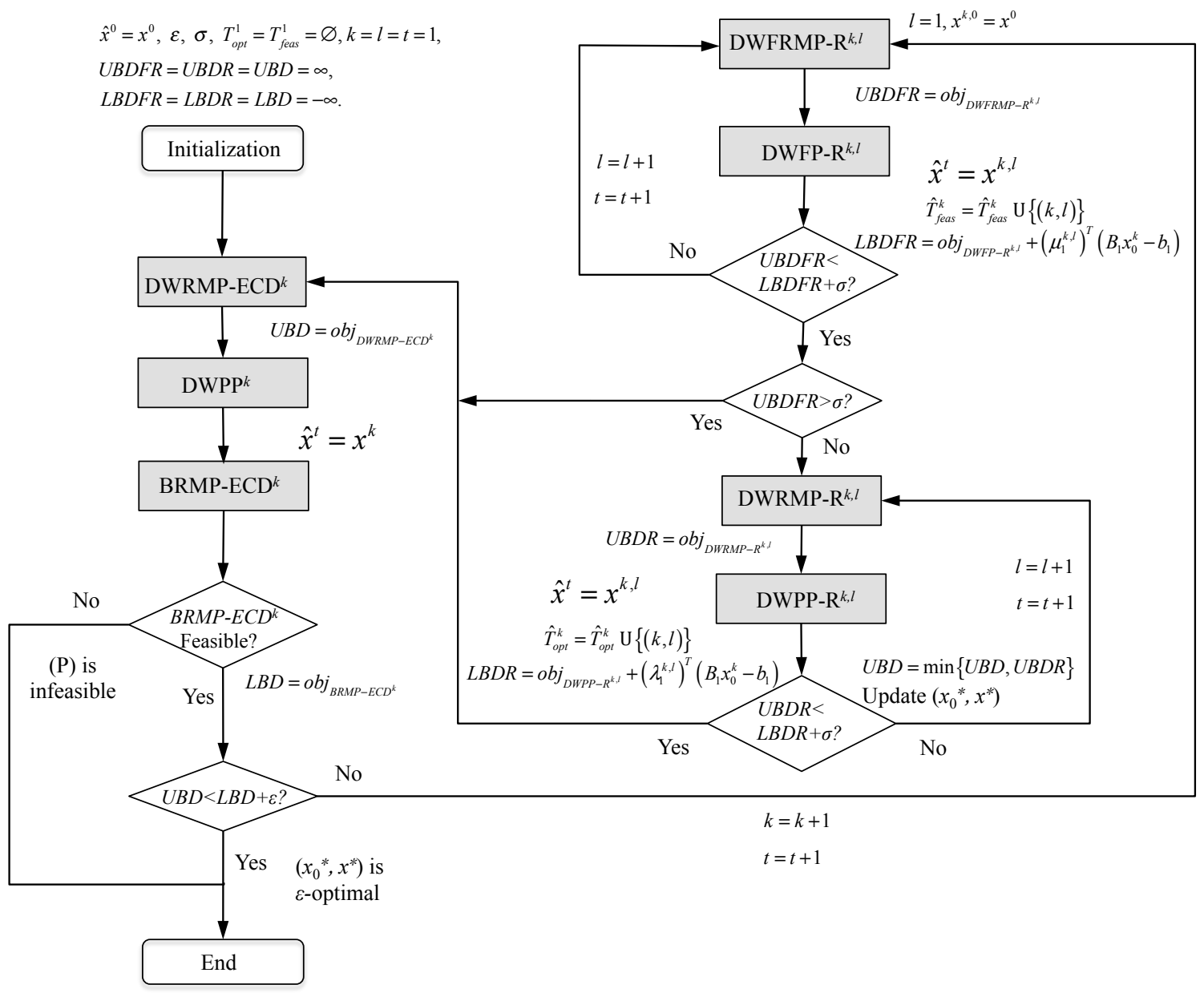

Figure 5: The flowchart of the extended cross decomposition method

$$
\begin{array}{ll} 
& \min _{x_{0}, \theta^{0}, \ldots, \theta^{k-1} \geq 0, z_{1} \geq 0, z_{1} \geq 0}\left\|z_{1}\right\|+\left\|z_{2}\right\| \\
\text { s.t. } & B_{1} x_{0}+A_{1} \sum_{i=0}^{t-1} \theta^{i} x^{i} \leq b_{1}+z_{1}, \\
& B_{2} x_{0}+A_{2} \sum_{i=0}^{t-1} \theta^{i} x^{i} \leq b_{2}+z_{2}, \\
& \sum_{i=0}^{t-1} \theta^{i}=1 \\
& x_{0} \in X_{0} .
\end{array}
$$



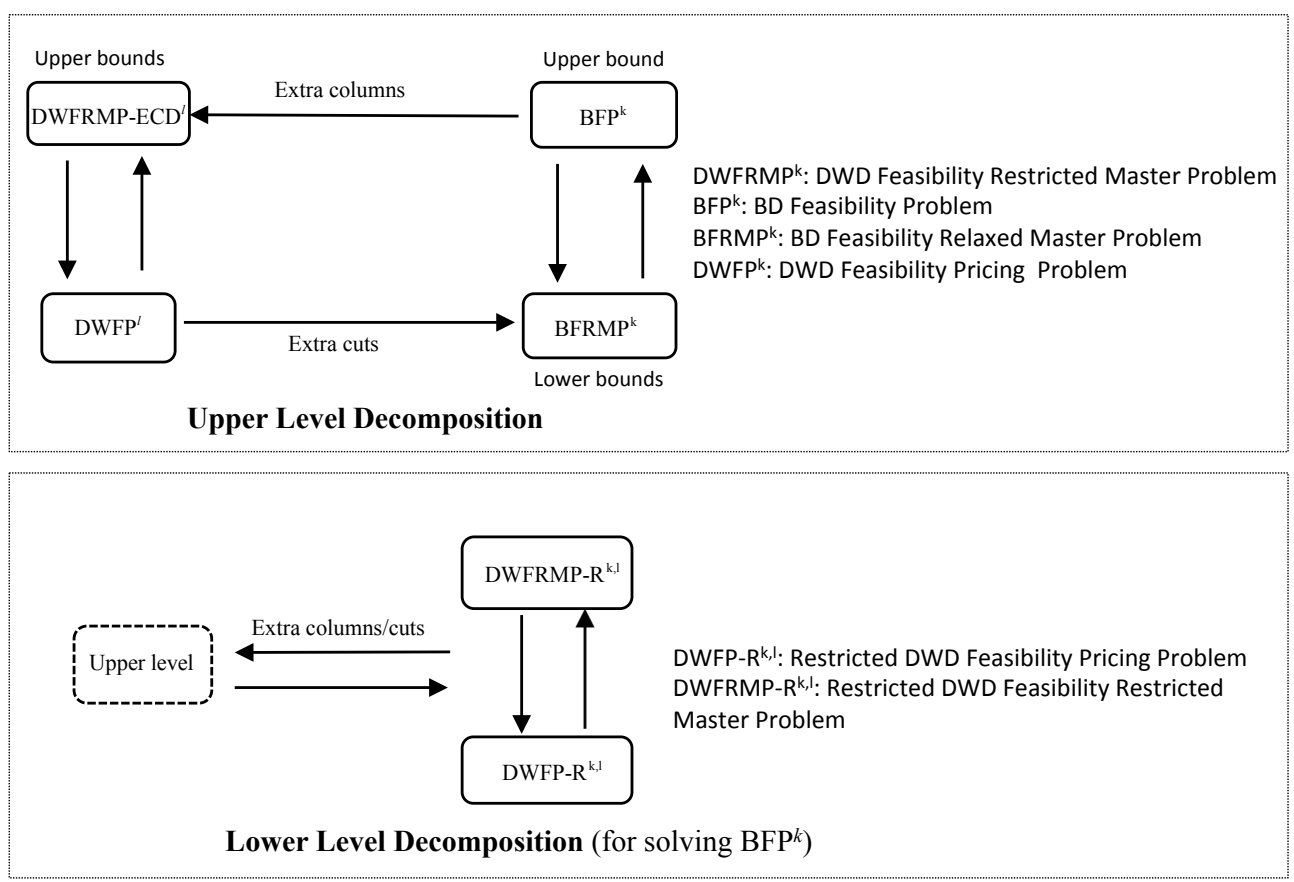

Figure 6: Diagram of the Phase I procedure for extended cross decomposition method

Let $\mu_{1}^{k}$ and $\mu_{2}^{k}$ be the Lagrange multipliers for the strong and weak linking constraints of the Problem (DWFRMP-ECD ${ }^{k}$ ), then the following DW pricing problem can be constructed and solved:

$$
\begin{aligned}
& \min _{x}\left(\mu_{1}^{k}\right)^{T} A_{1} x+\left(\mu_{2}^{k}\right)^{T} A_{2} x \\
& \text { s.t. } x \in X .
\end{aligned}
$$


The BD relaxed master problem solved in Phase I can be written as:

$$
\begin{array}{ll}
\min _{x_{0}, \eta} \eta & \\
\text { s.t. } \quad & \eta \geq o b j_{D W F P^{i}}+\left(\mu_{1}^{i}\right)^{\mathrm{T}}\left(B_{1} x_{0}-b_{1}\right)+\left(\mu_{2}^{i}\right)^{\mathrm{T}}\left(B_{2} x_{0}-b_{2}\right), \quad \forall i \in U_{\text {feas }}^{k}, \\
& \eta \geq o b j_{D W F P-R^{i, j}}+\left(\mu_{1}^{i, j}\right)\left(B_{1} x_{0}-b_{1}\right)+\left(\mu_{2}^{i, j}\right)^{\mathrm{T}} B_{2}\left(x_{0}-x_{0}^{i}\right), \quad \forall(i, j) \in \hat{T}_{\text {feas }}^{k}, \\
& x_{0} \in X_{0} .
\end{array}
$$

where $U_{\text {feas }}^{k}$ includes the indices of all previous iterations in which Problem $\left(\mathrm{DWFP}^{k}\right)$ is solved. Note that there are no feasibility cuts generated in Phase I, because Problem $\left(\mathrm{BFP}^{k}\right)$ is always feasible (provided $X$ and $X_{0}$ are nonempty).

Remark 1. The Phase I procedure illustrated in Figure 6 terminates finitely with an $\delta$ optimal solution of Problem (FP). If obj $j_{F P}>\delta$, then Problem (P) is infeasible; otherwise, Problem (P) is feasible and the optimal solution of Problem (FP) is a feasible solution of Problem (P).

In order for the optimality of Problem (FP) to precisely imply the feasibility of Problem (P), we need to select a sufficiently small optimality tolerance $\delta$ for Phase I (as least no larger than the feasibility tolerance of Problem (P)). The algorithmic flowchart of Phase I is similar to the one given in Figure 5, so it is omitted.

The optimal solution of Problem (FP) and all the other columns generated in Phase I are added to Problem (DWRMP-ECD ${ }^{k}$ ) in Phase II, so Problem $\left(\right.$ DWRMP-ECD $\left.^{k}\right)$ is always feasible. The cuts generated in Phase I can also be added to the BD relaxed master problem in Phase II, but these cuts will all appear as feasibility cuts, as explained in [21]. The Phase 
II BD relaxed master problem can be written as:

$$
\begin{array}{ll}
\min _{x_{0}, \eta} \eta & \\
\text { s.t. } \quad & \eta \geq o b j_{D W P P^{j}}+c_{0}^{T} x_{0}+\left(\lambda_{1}^{j}\right)^{\mathrm{T}}\left(B_{1} x_{0}-b_{1}\right)+\left(\lambda_{2}^{j}\right)^{\mathrm{T}}\left(B_{2} x_{0}-b_{2}\right) \quad \forall j \in U_{o p t}^{k}, \\
& \eta \geq o b j_{D W P P-R^{i, j}}+c_{0}^{T} x_{0}+\left(\lambda_{1}^{i, j}\right)^{\mathrm{T}}\left(B_{1} x_{0}-b_{1}\right)+\left(\lambda_{2}^{i, j}\right)^{\mathrm{T}} B_{2}\left(x_{0}-x_{0}^{i}\right), \quad \forall(i, j) \in \hat{T}_{o p t}^{k}, \\
& 0 \geq o b j_{D W F P^{i}}+\left(\mu_{1}^{i}\right)^{\mathrm{T}}\left(B_{1} x_{0}-b_{1}\right)+\left(\mu_{2}^{i}\right)^{\mathrm{T}}\left(B_{2} x_{0}-b_{2}\right), \quad \forall i \in U_{\text {feas }}^{k}, \\
& 0 \geq o b j_{D W F P-R^{i, j}}+\left(\mu_{1}^{i, j}\right)^{\mathrm{T}}\left(B_{1} x_{0}-b_{1}\right)+\left(\mu_{2}^{i, j}\right)^{\mathrm{T}} B_{2}\left(x_{0}-x_{0}^{i}\right), \quad \forall(i, j) \in \hat{T}_{\text {feas }}^{k}, \\
& x_{0} \in X_{0} .
\end{array}
$$

$\left(\right.$ BRMPII-ECD $\left.^{k}\right)$

\subsubsection{Adaptive switching between upper and lower level subproblems}

In [21], it is demonstrated that the CD method can be improved by switching between DWD and BD iterations in an adaptive way. The switching rules proposed in [21] can effectively avoid ineffective iterations at which columns and cuts generated do not help to close the optimality gap, so it can be adapted here to improve the convergence rate. Specifically, the ECD switching rules determine the alternation between the lower and the upper levels, following two criteria:

1. After an upper level DWD iteration, solve Problem $\left(\right.$ BRMPI-ECD $\left.^{k}\right)$ (for Phase I) or

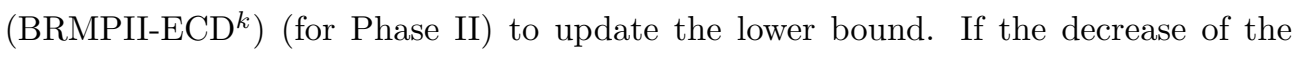
upper bound in the DWD iteration is more than the increase of the lower bound, then the algorithm will go to another DWD iteration. Otherwise, the algorithm will go to the lower level in order to solve $\left(\mathrm{BFP}^{k}\right)$ (for Phase I) or $\left(\mathrm{BPP}^{k}\right)$ (for Phase II).

2. After the convergence of a lower level procedure, solve Problem (DWFRMP-ECD ${ }^{k}$ ) (for Phase I) or (DWRMP-ECD ${ }^{k}$ ) (for Phase II). If the optimal objective value of the problem is better than the current upper bound, then the algorithm will go to an upper level DWD iteration. Otherwise, the algorithm will go to another BD iteration, which requires solving $\left(\mathrm{BFP}^{k}\right)$ (for Phase I) or $\left(\mathrm{BPP}^{k}\right)$ (for Phase II) via another lower level procedure. 
We tag the algorithm following the above rules as "ECD2" and the standard algorithm without the rules as "ECD1". The advantage of ECD2 will be seen through the case study.

\section{Application of ECD: Risk-averse two-stage stochastic programming}

\subsection{Background}

Different risk measurement metrics are adopted in the literature for risk-averse twostage stochastic programming, such as variance, variability index, probabilistic financial risk, downside risk, value-at-risk (VaR), etc [25]. In this paper, the conditional value-atrisk, CVaR, first proposed by Rockafellar and Uryasev [10] is used to measure risks because of its ability to account for the worse case scenario and computational tractability, and its close relationship to VaR [10] [26] [27] [28].

CVaR can be included in two-stage stochastic programming in two ways. One is to penalize CVaR in the objective function; in this case Problem (SP) does not have strong linking constraints, and it can be solved by classical decomposition methods. For example, Ahmed [29] used a cutting plane approach to solve a risk-averse stochastic linear program; the risk measured by a dispersion metric. Schultz and Tiedemann [30] applied Lagrangian decomposition to solve a risk averse stochastic mixed-integer programming problem. Noyan [7] developed two decomposition techniques based on generic BD to solve a stochastic LP minimizing CVaR. Qi et al. used multicut BD to solve a risk-averse problem [31].

The other way is to bound CVaR in constraints. Some applications with CVaR in constraints include portfolio optimization [10], oil and energy optimization [20], oil supply chain [32], large-scale industrial batch plants [33] [34], traveling saleman's problem [28]. Modeling CVaR as objective and as constraints have been shown by Krokhmal et al. to provide the same efficient frontier [26]. However, CVaR constrained approach is preferred because decision makers interpret and quantify right hand side easier than penalty parameters in the objective function, as argued by Fabian and Veszpremi [35]. The research on decomposition based strategy to solve CVaR constrained two-stage stochastic programming is very limited in the literature. Huang and Zheng [28] proposed a BD-type approach for the traveling saleman's problem with risk constraints. In their strategy, they seperated the risk constraints 
from scenario coupling constraints. Feasibility cuts were then developed to exclude solutions already generated. Bruno and Sagastizábal [20] developed two decomposition approaches to solve a two-stage stochastic linear program with CVaR constraints. The first approach is a Benders-like procedure, and the second approach is a bilevel decomposition procedure with Lagrangian decomposition in the upper level and BD in the lower level. The second approach is rigorous only for LP problems.

\subsection{CVaR-constrained two-stage stochastic programming}

We consider the following CVaR constraints for scenario-based risk-averse two-stage stochastic programming according to [26] [33] [34]:

$$
\begin{gathered}
\zeta+\frac{1}{(1-\beta)} \sum_{\omega \in S} p_{\omega} \psi_{\omega} \leq b_{0}, \\
\psi_{\omega} \geq f_{\omega}\left(x_{\omega}\right)-\zeta, \quad \psi_{\omega} \geq 0, \quad \forall \omega \in\{1, \ldots, s\},
\end{gathered}
$$

where $f_{\omega}\left(x_{\omega}\right)$ denotes a scenario dependent loss function of the second-stage variables at scenario $\omega, p_{\omega} \in[0,1]$ denotes the probability of scenario $\omega\left(\sum_{\omega=1}^{s} p_{\omega}=1\right), \beta \in[0,1]$ is a user specified probability, $b_{0}$ is a user specified risk threshold for the loss function over all scenarios. When the CVaR constraints are included, the two-stage stochastic programming problem can be written as:

$$
\begin{aligned}
& \min _{\substack{x_{0}, x_{1}, \ldots, x_{s} \\
\zeta, \psi_{1}, \ldots, \psi_{s}}} \sum_{\omega=1}^{s}\left(c_{0, \omega}^{\mathrm{T}} x_{0}+c_{\omega}^{\mathrm{T}} x_{\omega}\right) \\
& \text { s.t. } A_{0, \omega} x_{0}+A_{\omega} x_{\omega} \leq b_{0, \omega}, \quad \omega \in\{1, \ldots, s\}, \\
& \quad \psi_{\omega} \geq f_{\omega}\left(x_{\omega}\right)-\zeta, \quad \omega \in\{1, \ldots, s\}, \\
& \quad \zeta+\sum_{\omega=1}^{s} \hat{p}_{\omega} \psi_{\omega} \leq b_{0}, \\
& \quad x_{\omega} \in X_{\omega}, \psi_{\omega} \geq 0, \quad \forall \omega \in\{1, \ldots, s\}, \\
& x_{0} \in X_{0}, \zeta \in \mathbb{R},
\end{aligned}
$$


271

so a valid upper bound on $\zeta$ is $\zeta^{u p}=b_{0}$

Next, we show that a valid lower bound on $\zeta$ is

$$
\zeta^{l o}=\min \left\{-\frac{(1-\beta)}{\beta} b_{0}+\frac{1}{\beta} f^{l o}, f^{l o}\right\},
$$

where $f^{l o}$ is a valid lower bound for the loss function for any scenario, i.e.,

$$
f^{l o} \leq \min _{\omega \in\{1, \cdots, s\}}\left\{\min _{x_{\omega} \in X_{\omega}} f_{\omega}\left(x_{\omega}\right)\right\} .
$$

We show this by showing that any $\zeta$ that is less than $\zeta^{l o}$ does not satisfy (3). This is because 
$\forall \zeta<\zeta^{l o}$,

$$
\begin{aligned}
\zeta+\frac{1}{1-\beta} \sum_{\omega=1}^{s} p_{\omega} \psi_{\omega} & \geq \zeta+\frac{1}{1-\beta} \sum_{\omega=1}^{s} p_{\omega}\left(f_{\omega}\left(x_{\omega}\right)-\zeta\right) \\
& \geq \zeta+\frac{1}{1-\beta} \sum_{\omega=1}^{s} p_{\omega}\left(f^{l o}-\zeta\right) \\
& =\zeta+\frac{\left(f^{l o}-\zeta\right)}{1-\beta} \sum_{\omega=1}^{s} p_{\omega} \\
& =\zeta+\frac{\left(f^{l o}-\zeta\right)}{1-\beta} \\
& =\frac{-\beta}{1-\beta} \zeta+\frac{1}{1-\beta} f^{l o} \\
& >\frac{-\beta}{1-\beta}\left\{-\frac{(1-\beta)}{\beta} b_{0}+\frac{1}{\beta} f^{l o}\right\}+\frac{1}{1-\beta} f^{l o} \\
& =b_{0} .
\end{aligned}
$$

Finally, we show that $\psi_{\omega}^{u p}=f_{\omega}^{u p}-\zeta^{l o}$ is a valid upper bound for $\psi_{\omega}$, where $f_{\omega}^{u p}$ is a valid upper bound for the loss function for scenario $\omega$, i.e.,

$$
f_{\omega}^{u p} \geq \max _{x_{\omega} \in X_{\omega}} f_{\omega}\left(x_{\omega}\right)
$$

According to equation (4), this can be shown by showing that $f_{\omega}^{u p}-\zeta^{l o} \geq 0$, and $f_{\omega}^{u p}-\zeta^{l o} \geq$ $f_{\omega}\left(x_{\omega}\right)-\zeta$, and these two relations result directly from the definitions of $f_{u p}^{\omega}$ and $\zeta^{l o}$.

In summary, the bounds of $\zeta$ and $\psi_{\omega}$ can be computed as follows:

$$
\begin{aligned}
& \psi_{\omega}^{l o}=0, \quad \forall \omega \in\{1, \cdots, s\}, \\
& \zeta^{u p}=b_{0}, \\
& \zeta^{l o}=\min \left\{-\frac{(1-\beta)}{\beta}+\frac{1}{\beta} f^{l o}, f^{l o}\right\}, \\
& \psi_{\omega}^{u p}=f_{\omega}^{u p}-\zeta^{l o}, \quad \forall \omega \in\{1, \cdots, s\} .
\end{aligned}
$$

The ECD subproblems for Problem (CVaR-SP), including those for Phase I and Phase II, are explained in Appendix A. 


\section{Case Studies}

We compare four solution methods for solving risk-averse stochastic programming problems in the case studies. These methods are monolith (i.e., solving the full problem using a commercial optimization solver), BLD (i.e., the bilevel decomposition method proposed in Section 2), ECD1 (the proposed ECD method without adaptive switching between the two levels), and ECD2 (the proposed ECD method with adaptive switching between the two levels). The simulation was run on a virtual machine setup running Ubuntu 16.04 with a 2.4 GHz CPU and 4 GB of memory. The two formulations and the solution methods were programmed on GAMS 24.6.1 [36], with CPLEX 12.6.3 [37] being the LP/MILP solver. GUSS [38], a GAMS extension, was utilized in all decomposition methods (with default GUSS options), in order to achieve efficient model generation and solution for the decomposed scenario problems. For all decomposition methods the norm functions in the subproblems are the 1-norm. The relative optimality tolerance used for all solution methods was $10^{-3}$. Since BLD needs initial values for the first-stage decisions to start the algorithm, we set the values be zero for all problem instances.

\subsection{Case $A$ \\ 5.1.1. Problem Description and Advantage of Risk-Averse Formulation}

The case study considers an energy and bio-product supply chain optimization (SCO) problem originally studied in [39]. The supply chain considered has four layers involving material collecting, material preprocessing, energy and bio-product production and product distribution. The goal of the SCO is to determine the optimal configuration of the supply chain network and the technologies used in the production plants, such that the total profit is maximized and the customer demands at the demand locations are satisfied. McLean and Li [40] developed a two-stage stochastic MILP formulation for the SCO problem, where the first-stage decisions are binary variables determining whether or not specific units are to be developed and specific technologies are to be adopted for energy/bio-product production, and the second-stage decisions are continuous variables determining material and product flows in the operation of the supply chain. 
The two-stage stochastic MILP in [40] does not contain strong linking constraints, so it is not suitable for evaluating the decomposition methods developed in this paper. Therefore, we modify the problem by adding a CVaR constraint. In order to do this, we first assume that the demand uncertainty is in the minimum electricity demands rather than the maximum demands. We also assume that the supply chain needs to satisfy uncertain minimum electricity demand for "most scenarios" (rather than for all scenarios), so the minimum demand requirement can be expressed as a CVaR constraint, where the loss function $f_{\omega}\left(x_{\omega}\right)$ is the total unsatisfied minimum electricity demands (i.e., the total minimum electricity demand minus the total electricity supply). The minimum electricity demand (at each demand location) and the yield of corn stover are the two uncertain parameters considered in the model, which are assumed to be uniformly distributed in ranges $\left[\begin{array}{ll}1 & 3\end{array}\right] 10^{6} \cdot M W h / y e a r$ and $[790890] \mathrm{t} / \mathrm{km}^{2} \cdot$ year, respectively. The model involves 18 binary variables and $2376 \mathrm{~s}$ +7 continuous variables (where $s$ refers to the number of scenarios). Other details of the model are provided in the supplemental materials.

In order to show the advantage of including the CVaR constraints in the formulation, we compare in Table 1 the quality of the solutions obtained from formulations with and without the CVaR constraints. 169 scenarios are addressed in each of the formulations. It can be seen that, when no CVaR constraints are included in the formulation, all preprocessing centers $\left(m_{1}, \cdots, m_{6}\right)$ except $m_{3}$ are to be developed, and main plant $n_{2}$ only includes drygrind facilities. When CVaR constraints are included in the formulation, the preprocessing center that is not developed is $m_{4}$ instead of $m_{3}$, and main plant $n_{2}$ also needs incineration facilities in order to generate more electricity to meet the (uncertain) minimum demands for electricity. As a result, the solution from the risk-averse formulation is more robust in terms of observing the minimum electricity demand constraint (although it leads to a lower expected profit), as indicated by the lower CVaR risk measure and the higher probability of minimum electricity demand satisfaction. Note that the CVaR constraints do not enforce $100 \%$ demand satisfaction. This is different from using "hard" demand constraints that require demand satisfaction for all uncertainty realizations (which is usually overly conservative). 
Table 1: Comparison of solutions from the two stochastic programming formulations - Case A

\begin{tabular}{|c|c|c|}
\hline Formulation (169 scenarios) & Without CVaR constraints & With CVaR constraints \\
\hline Preprocessing centers & $m_{1}, m_{2}, m_{4}, m_{5}, m_{6}$ & $m_{1}, m_{2}, m_{3}, m_{5}, m_{6}$ \\
\hline \multirow[t]{7}{*}{ Facilities in main plant } & $n_{1}:$ Dry-grind & $n_{1}:$ Dry-grind \\
\hline & Incineration & Incineration \\
\hline & $n_{2}:$ Dry-grind & $n_{2}:$ Dry-grind \\
\hline & & Incineration \\
\hline & $n_{3}:$ Dry-grind & $n_{3}:$ Dry-grind \\
\hline & Sawing & Sawing \\
\hline & Incineration & Incineration \\
\hline $\begin{array}{l}\text { Predicted expected profit } \\
(\text { Million } \$)\end{array}$ & 29.43 & 26.96 \\
\hline $\mathrm{CVaR}\left(\times 10^{3} \mathrm{MWh} /\right.$ year $) \dagger$ & 273.76 & -13.73 \\
\hline $\begin{array}{l}\text { Probability of minimum electricity } \\
\text { demands satisfied } \ddagger\end{array}$ & $53.3 \%$ & $97.3 \%$ \\
\hline
\end{tabular}

${ }^{\dagger}$ : CVaR of the first-stage decisions, estimated by sampling 225 realizations of uncertainty (i.e., 15 samples for each uncertain parameter).

¥: The probabilities were also estimated from the 225 realizations of uncertainty.

\subsubsection{Computational Results and Discussions}

Tables 2, 3, 4 and 5 show the computational results of the four solution approaches. We can see that the monolith approach is the fastest for the problem with 9 scenarios, but its solution time grows rapidly with the number of scenarios. On the other hand, the solution times of the decomposition approaches increase relatively slowly with the number of scenarios, because the number of iterations of these approaches do not increase significantly with the number of scenarios. Therefore, when the number of scenario is larger than 49 , all the decomposition approaches are faster than the monolith approach (by 100\% to more than an order of magnitude). We can also see that the solution time of each decomposition method is dominated by the time for solving all pricing subproblems, which depends on the total number of upper level and lower level DWD iterations. Compared to BLD, ECD1 requires fewer upper level iterations and therefore fewer total DWD iterations, because its upper level is CD which converges faster than BD (except for the 121 scenario instance). So ECD1 is mostly faster than BLD. Furthermore, ECD2 is consistently faster than BLD and mostly faster than ECD1. This is because ECD2 can avoid ineffective upper level DWD 
iterations and it requires fewer upper level iterations.

Note that "total solver time" in the tables stands for the sum of solution times of all subproblems solved in a decomposition method, and "wall time" in the tables stands for the actual time used by a decomposition method (including the total solver time and all computing overhead). The large discrepancy between the total solver time and the wall time came from the fact that the data exchange (between the subproblems) on GAMS was inefficient. If the methods are implemented using $\mathrm{C}++$ that allows more efficient data exchange, the wall times will be more close to the total solver times.

The advantage of ECD2 is also demonstrated by Figures 7 and 8 . Figure 7 illustrates how the upper and lower bounds change with the upper level iterations (but not with lower level iterations at which the bounds do not change). Figure 8 shows the bound curves after iteration 24 with an adjusted scale (so that the tails of the curves are more clear). We can see that, in the first several iterations, the optimality gap of ECD2 is not as small as that of BLD, but as the algorithm progresses, the upper and lower bounds from ECD2 approach each other more quickly than those from BLD do. So compared to BLD, ECD2 converges to an optimal solution in fewer upper level iterations (for all problem instances). ECD1 also requires fewer iterations compared to BLD for most problem instances. It should be noted that, while $10^{-3}$ was set as the relative optimality tolerances in the case study, the figures also indicate the convergence rates with difference tolerances. For example, if the relative optimality tolerance is decreased to $10^{-4}$, the solution times of ECD1 and ECD2 will not change much but the solution time of BLD will be much longer. But if the relative optimality tolerance is increased to $10^{-2}$, BLD will terminate more quickly than ECD1 while ECD2 will still be the fastest. If the relative optimality tolerance is as large as $10^{-1}$, BLD will terminate within 20 iterations for all problem instances and becomes the fastest method. 
Table 2: Computational results of Case A - Monolith (Unit for time: sec)

\begin{tabular}{lcccccc}
\hline Number of scenarios & 9 & 25 & 49 & 81 & 121 & 169 \\
\hline Optimal obj. (Million $\$$ ) & -26.96 & -26.96 & -26.96 & -26.96 & -26.96 & -26.96 \\
Wall time & 118 & 2563 & 4971 & 37851 & 55117 & $-\ddagger$ \\
\hline \multicolumn{1}{c}{ Table 3: Computational results of Case A - BLD (Unit for time: sec) } & \\
\hline Number of scenarios & 9 & 25 & 49 & 81 & 121 & 169 \\
\hline Number of upper BD iterations & 56 & 74 & 76 & 103 & 55 & 152 \\
Number of lower DWD iterations & 245 & 310 & 364 & 491 & 236 & 741 \\
Optimal obj. (Million \$) & -26.96 & -26.94 & -26.96 & -26.96 & -26.96 & -26.96 \\
Time for pricing problems & 232 & 986 & 2156 & 5132 & 2689 & 18006 \\
Time for DW restricted MP & 3.4 & 8.21 & 18.9 & 43.5 & 37.9 & 154 \\
Time for BD relaxed MP & 1.8 & 2.41 & 1.45 & 1.83 & 0.63 & 3.4 \\
Total solver time & 238 & 996 & 2176 & 5177 & 3728 & 18164 \\
Wall time & 392 & 1320 & 2991 & 8224 & 5012 & 40184 \\
\hline
\end{tabular}

Table 4: Computational results of Case A - ECD1 (Unit for time: sec)

\begin{tabular}{lcccccc}
\hline Number of scenarios & 9 & 25 & 49 & 81 & 121 & 169 \\
\hline Num. of upper BD iterations & 53 & 87 & 67 & 65 & 89 & 75 \\
Num. of upper DWD iterations & 27 & 44 & 34 & 33 & 45 & 38 \\
Num. of lower DWD iterations & 109 & 186 & 160 & 140 & 189 & 154 \\
Num. of pricing problems solved \# & 136 & 230 & 194 & 173 & 234 & 192 \\
Optimal obj. (Million \$) & -26.94 & -26.95 & -26.96 & -26.96 & -26.96 & -26.93 \\
Time for pricing problems & 123 & 707 & 1114 & 1738 & 3924 & 4501 \\
Time for DW restricted MP & 6.1 & 49.7 & 73.4 & 83.3 & 335.6 & 271 \\
Time for BD relaxed MP & 0.5 & 1.6 & 0.57 & 0.45 & 0.79 & 0.6 \\
Total solver time & 130 & 759 & 1188 & 1840 & 4260 & 4774 \\
Wall time & 205 & 1026 & 1543 & 2368 & 5790 & 6336 \\
\hline \multicolumn{1}{c}{ Table 5: Computational results of Case A ECD2 (Unit for time: sec) } & \\
\hline Number of scenarios & 9 & 25 & 49 & 81 & 121 & 169 \\
\hline Num. of upper BD iterations & 29 & 36 & 42 & 52 & 43 & 36 \\
Num. of upper DWD iterations & 4 & 4 & 4 & 4 & 4 & 4 \\
Num. of lower DWD iterations & 108 & 137 & 157 & 216 & 166 & 133 \\
Num. of pricing problems solved \# & 112 & 141 & 161 & 220 & 170 & 137 \\
Optimal obj. (Million \$) & -26.96 & -26.94 & -26.96 & -26.94 & -26.94 & -26.96 \\
Time for pricing problems & 103 & 425 & 813.1 & 2103.3 & 2611 & 2669 \\
Time for DW restricted MP & 6.7 & 29.3 & 55.5 & 181.4 & 159 & 116 \\
Time for BD relaxed MP & 0.3 & 0.48 & 0.5 & 1.2 & 0.58 & 0.4 \\
Total solver time & 111 & 455 & 869 & 2302 & 2771 & 2786 \\
Wall time & 187 & 632 & 1171 & 3115 & 3783 & 3924 \\
\hline
\end{tabular}

\#: Total number of IPP, DWPP, DWFP, DWPP-R and DWFP-R solved

¥: CPLEX found an MIP solution after 18 hours but could not solve final LP within 24 hours 

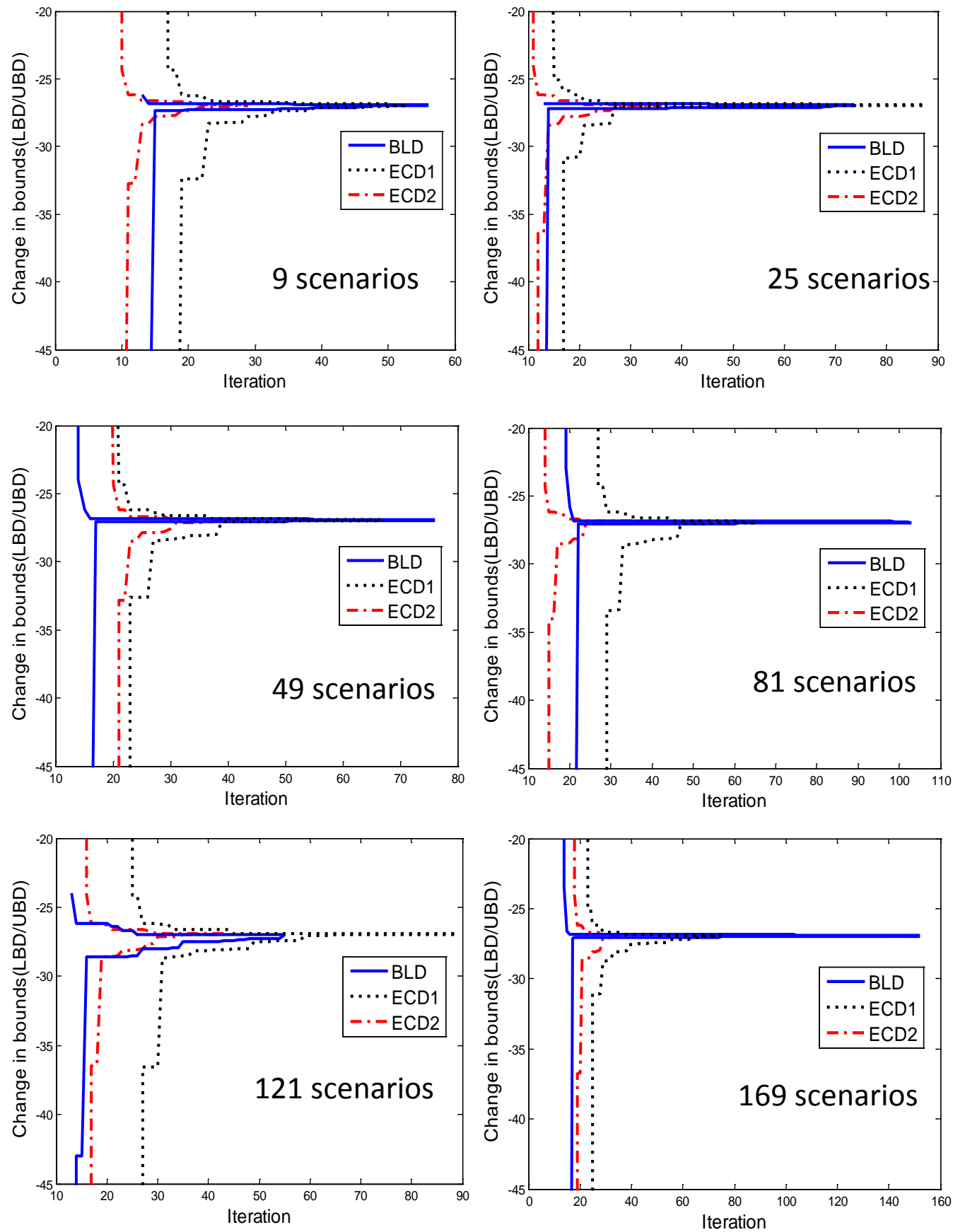

Figure 7: Comparison of bound evolution in different decomposition methods - Case A 

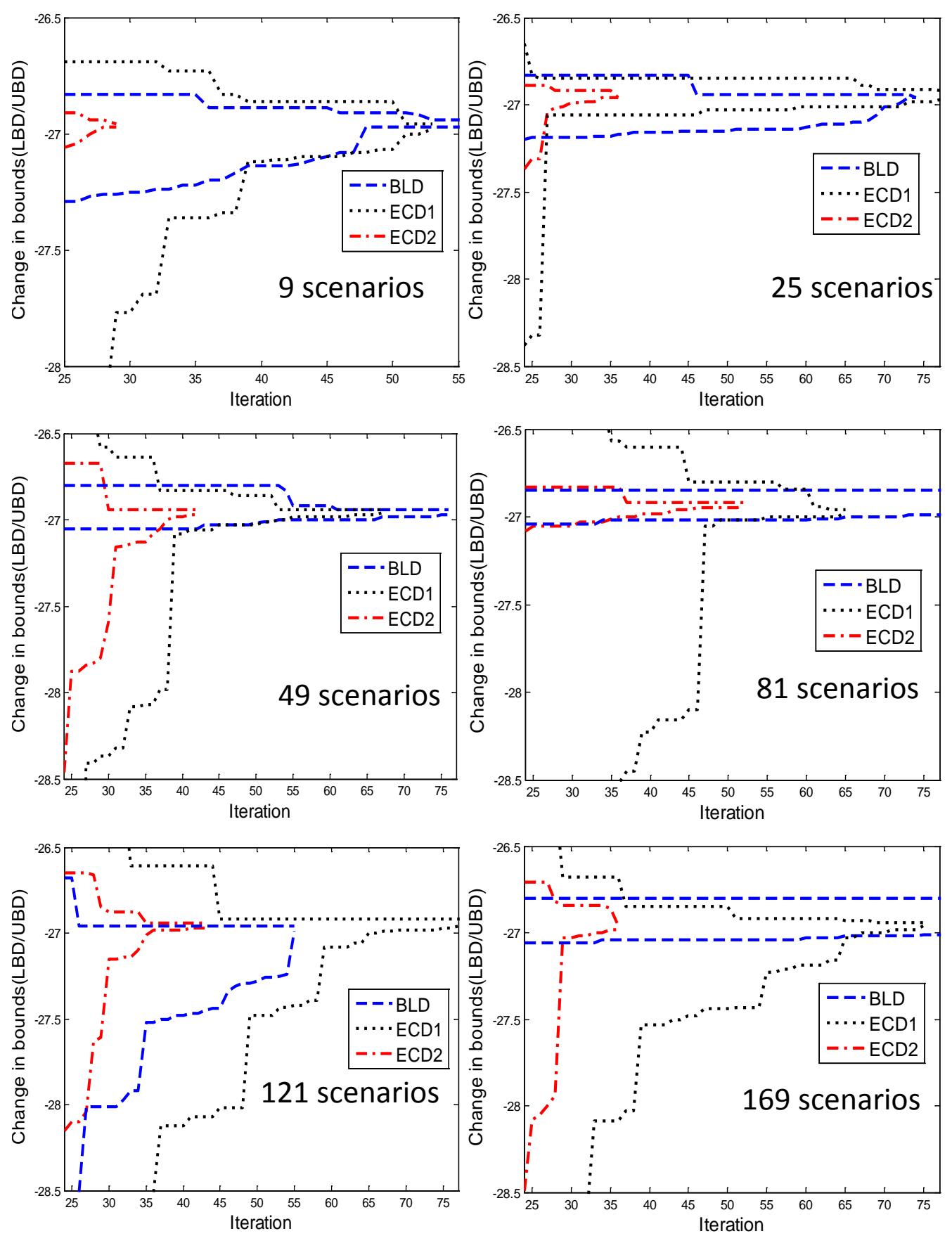

Figure 8: Comparison of bound evolution in different methods - Case A (after iteration 25) 
5.2. Case $B$

\subsubsection{Problem Description and Advantage of Risk-Averse Formulation}

The case study considers an industrial chemical supply chain management problem that was originally studied and formulated as a two-stage stochastic MILP in [41]. The supply chain involves 5 manufacturing plants that produce 23 grades of final products from 55 grades of raw materials, a number of warehouses and distribution centers that stores raw materials and final products, and 5 regional markets. The goal of the SCO problem is to determine the optimal capacities of the 5 manufacturing plants such that the total profits are maximized and the minimum demands at the regional markets are satisfied. The first-stage decisions are the capacities of plants (represented by integer variables), and the secondstage decisions are material or product flow rates (represented by continuous variables), which reflect how the supply chain is operated.

Again, in this paper, we revise the two-stage stochastic MILP into a risk-averse two-stage stochastic MILP. In order to do this, we introduce target demands for the regional markets, which are uncertain and larger than the minimum demands. We add a CVaR constraint that requires the target demands be satisfied for "most scenarios". The loss functions in the CVaR constraint is the largest unsatisfied target demand over all regional markets. The two uncertain parameters addressed in the formulation represent the uncertain target demands at the regional markets, as defined in [41], and the minimum demands are set to be $70 \%$ of the target demands. The MILP model involves 5 positive integer variables and $8210 \mathrm{~s}+6$ continuous variables (where $s$ refers to the number of scenarios). More details of the model are provided in the supplemental materials.

The advantage of the risk-averse formulation is demonstrated by the results in Table 6 . Without the CVaR constraints, the formulation seeks to maximize the expected profit but does not ensure that all target demands are satisfied. As a result, the total plant capacity determined by this stochastic programming formulation is smaller than that determined by the risk-averse formulation. While the risk-neutral formulation predicts a higher expected profit (because of lower plant investment costs), it leads to a low target demand satisfaction probability $(25 \%)$. On the other hand, the plant capacities determined by the risk-averse 
Table 6: Comparison of solutions from the two stochastic programming formulations - Case B

\begin{tabular}{lll}
\hline Formulation (64 scenarios) & Without CVaR constraints & With CVaR constraints \\
\hline Plant Capacities (units/year) & Plant 1: 5400 & Plant 1: 5100 \\
& Plant 2: 1200 & Plant 2: 1800 \\
& Plant 3: 600 & Plant 3: 900 \\
& Plant 4: 1200 & Plant 4: 1500 \\
& Plant 5: 3900 & Plant 5: 3600 \\
\hline $\begin{array}{l}\text { Predicted expected profit } \\
\text { (Billion } \$)\end{array}$ & 22.28 & 20.23 \\
\hline $\begin{array}{l}\text { Probability of target demand } \\
\text { satisfaction }\end{array}$ & $25.8 \%$ & $100 \%$ \\
\hline $\begin{array}{l}\dagger \\
\text { uncertain parameter). }\end{array}$ & \\
\hline
\end{tabular}

formulation enable larger total production rates, which can almost always satisfy all target demands. Note that the $100 \%$ demand satisfaction rate was estimated from the 225 uncertainty realizations, which does not stand for the true probability.

\subsubsection{Computational Results and Discussion}

We summarize the computational results of the risk-averse formulation for Case Study B in Tables 7-10. Again, we see that the monolith approach is efficient when the number of scenarios is small, but its solution time grows rapidly with the number of scenarios. On the other hand, the solution times of the three decomposition methods grows almost linearly with the number of scenarios, because the numbers of the iterations needed by the methods remain almost the same for different numbers of scenarios. In addition, for each decomposition method, most part of the solution time is devoted to pricing problems involved in upper/lower level DWD iterations. As a result, ECD2 is better than BLD and ECD1 (as it involves fewer DWD iterations), and BLD is slightly better than ECD1 (as the two methods have similar number of iterations but ECD1 spends additional time on DW restricted master problems).

Figure 9 compares how the bounds of the decomposition methods change with the upper level iterations for different scenario instances. We can see that for case B, BLD does not generate good upper and lower bounds at the beginning of the solution procedures but 
${ }_{431}$ ECD1 and ECD2 do. However, ECD1 and ECD2 have different rates of convergence, so 432 ECD1 is slightly slower than BLD and ECD2 is faster. Overall, all the three methods have 433 quite fast rates of convergence and ECD2 is the fastest. In addition, ECD2 will remain the ${ }_{434}$ fastest algorithm if the tolerance is increased or decreased by an order of magnitude. 
Table 7: Computational Results of Case B - Monolith (Unit for time: sec)

\begin{tabular}{lcccccc}
\hline Number of scenarios & 9 & 16 & 25 & 36 & 49 & 64 \\
\hline Optimal obj. (Billion \$) & -20.20 & -20.22 & -20.22 & -20.22 & -20.22 & -20.23 \\
Wall time & 1288 & 3570 & 6521 & 11121 & 11640 & 20947 \\
\hline \multicolumn{1}{c}{ Table 8: Computational Results of Case B - BLD (Unit for time: sec) } & \\
\hline Number of scenarios & 9 & 16 & 25 & 36 & 49 & 64 \\
\hline Number of upper BD iterations & 57 & 52 & 56 & 59 & 67 & 74 \\
Number of lower DWD iterations & 142 & 148 & 152 & 169 & 184 & 187 \\
Optimal obj. (Billion \$) & -20.20 & -20.22 & -20.22 & -20.22 & -20.22 & -20.23 \\
Time for pricing problems & 653 & 1095 & 1694 & 2499 & 3502 & 4369 \\
Time for DW restricted MP & 5.86 & 13.3 & 24.9 & 45.36 & 63.4 & 118.6 \\
Time for BD relaxed MP & 2.37 & 1.7 & 2.92 & 2.77 & 0.93 & 0.97 \\
Total solver time & 661 & 1110 & 1721 & 2547 & 3566 & 4488 \\
Wall time & 1400 & 1868 & 2710 & 3903 & 4721 & 5777 \\
\hline
\end{tabular}

Table 9: Computational Results of Case B - ECD1 (Unit for time: sec)

\begin{tabular}{lcccccc}
\hline Number of scenarios & 9 & 16 & 25 & 36 & 49 & 64 \\
\hline Num. of upper BD iterations & 65 & 71 & 71 & 77 & 75 & 77 \\
Num. of upper DWD iterations & 32 & 35 & 35 & 38 & 37 & 38 \\
Num. of lower DWD iterations & 128 & 140 & 137 & 145 & 137 & 143 \\
Num. of pricing problems solved \# & 160 & 175 & 172 & 183 & 174 & 181 \\
Optimal obj. (Billion \$) & -20.20 & -20.22 & -20.22 & -20.22 & -20.22 & -20.23 \\
Time for pricing problems & 582 & 1056 & 1545 & 2243 & 2706 & 4501 \\
Time for DW restricted MP & 30.8 & 103.7 & 207 & 440 & 749 & 1245 \\
Time for BD relaxed MP & 0.43 & 0.4 & 0.45 & 0.3 & 0.33 & 0.4 \\
Total solver time & 613 & 1160 & 1753 & 2684 & 3456 & 4703 \\
Wall time & 1335 & 2074 & 2798 & 4040 & 5078 & 6445 \\
\hline
\end{tabular}

Table 10: Computational Results of Case B - ECD2 (Unit for time: sec)

\begin{tabular}{lcccccc}
\hline Number of scenarios & 9 & 16 & 25 & 36 & 49 & 64 \\
\hline Num. of upper BD iterations & 37 & 40 & 40 & 43 & 42 & 43 \\
Num. of upper DWD iterations & 4 & 4 & 4 & 4 & 4 & 4 \\
Num. of lower DWD iterations & 96 & 104 & 107 & 109 & 108 & 110 \\
Num. of pricing problems solved \# & 100 & 108 & 111 & 113 & 112 & 114 \\
Optimal obj. (Billion \$) & -20.20 & -20.22 & -20.22 & -20.22 & -20.22 & -20.23 \\
Time for pricing problems & 447 & 791 & 1165 & 1609 & 2082 & 2554 \\
Time for DW restricted MP & 46.2 & 86.5 & 184 & 370 & 589 & 1028 \\
Time for BD relaxed MP & 0.24 & 0.28 & 0.28 & 0.32 & 0.3 & 0.3 \\
Total solver time & 493 & 878 & 1349 & 1980 & 2671 & 3582 \\
Wall time & 956 & 1515 & 2096 & 2937 & 3891 & 4938 \\
\hline
\end{tabular}

\#: Total number of IPP, DWPP, DWFP, DWPP-R and DWFP-R solved 

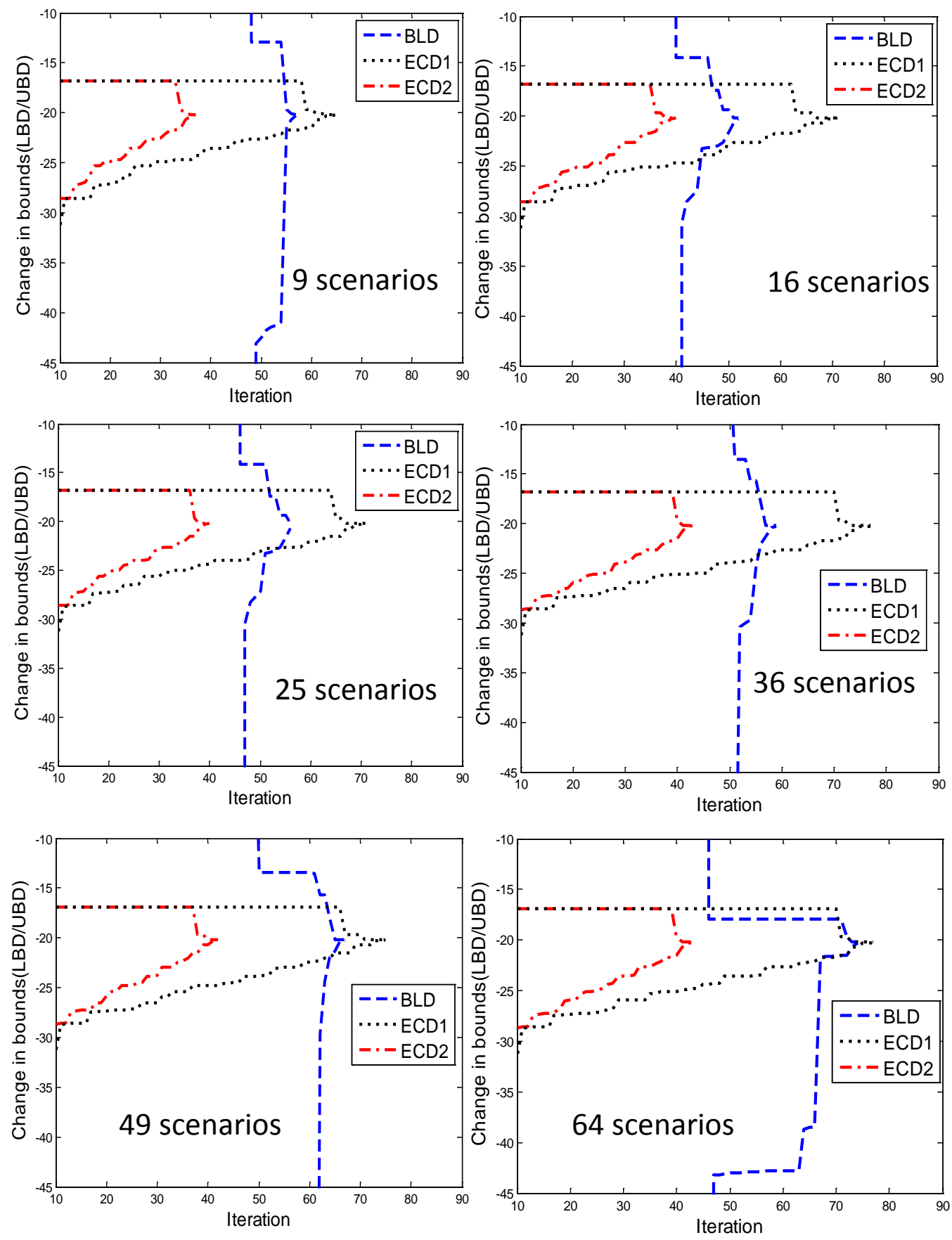

Figure 9: Comparison of bound evolution in different methods - Case B 
5.3. Case $C$

In the last two case study problems, we see how the different solution methods perform as the number of scenarios, or the number of continuous variables and constraints, increase. In this case study, we examine how the solution methods perform as the candidate integer decisions increase. We generate 6 new problem instances from the 25-scenario problem instance in Case B. In case B, the capacities of the 5 plants are multiples of 300 (ton per unit time); in other words, the interval of two consecutive candidate plant capacities is 300. In this case, the capacity intervals in the 6 new problem instances are 50, 150, 250, $350,450,550$, respectively. With the increase of capacity interval, the number of candidate plant capacities decreases, and the resulting MILP tends to be easier (in spite of the same problem size), because the number of nodes to be explored in branch-and-bound search or the number of iterations in decomposition tends to reduce.

The performance of the different solution methods for the 6 problem instances is shown in Tables 11-14. In Table 11 that summarizes the results of the monolith approach, we can see that the problem tends to require more solution time when the capacity interval decreases (from the rightmost column toward the left). This trend is more clear and consistent in Tables 12-14 where the decomposition methods are applied. Generally, the decomposition methods are more efficient than the monolith approach (with an exception that ECD1 is slower than the monolith approach when the capacity interval is 50). Among the three decomposition methods, ECD1 is the slowest, because it needs the most number of iterations to converge. When the problem is relatively easy (i.e., the capacity interval is relatively large), BLD is better than ECD2; otherwise, ECD2 is better. This is because when the problem is easy, the number of iterations BLD needs to converge is already small (e.g. only $44 \mathrm{BD}$ iterations for the rightmost problem instance); in this situation, integrating the extra DWD procedure into BLD does not reduce much number of iterations but requires solving more subproblems. The performance of the decomposition methods is also compared in Figure 10 in terms of how the bounds change over the iterations. Obviously, ECD2 converges the fastest for all problem instances, and ECD1 converges the slowest. The convergence rates of all decomposition methods tend to decrease when the capacity interval increases. 
Table 11: Computational Results of Case C - Monolith (Unit for time: sec)

\begin{tabular}{lcccccc}
\hline Capacity interval (ton/time) & 50 & 150 & 250 & 350 & 450 & 550 \\
\hline Optimal obj. (Billion \$) & -20.32 & -20.22 & -19.67 & -19.56 & -19.66 & -19.63 \\
Wall time & 4458 & 5120 & 4287 & 4654 & 4094 & 3438 \\
\hline \multicolumn{1}{c}{ Table 12: Computational Results of Case C - BLD (Unit for time: sec) } & \\
\hline Capacity interval (ton/time) & 50 & 150 & 250 & 350 & 450 & 550 \\
\hline Number of upper BD iterations & 103 & 74 & 71 & 48 & 54 & 44 \\
Number of lower DWD iterations & 244 & 187 & 185 & 127 & 139 & 113 \\
Optimal obj. (Billion \$) & -20.32 & -20.22 & -19.68 & -19.56 & -19.66 & -19.63 \\
Time for pricing problems & 2843 & 2188 & 2216 & 1482 & 1661 & 1322 \\
Time for DW restricted MP & 37.8 & 32.3 & 35.5 & 23.6 & 20.3 & 22.6 \\
Time for BD relaxed MP & 1.4 & 0.7 & 1.2 & 0.6 & 0.7 & 0.8 \\
Total solver time & 2882 & 2221 & 2253 & 1507 & 1682 & 1345 \\
Wall time & 3942 & 3139 & 3151 & 2153 & 2367 & 1916 \\
\hline
\end{tabular}

Table 13: Computational Results of Case C - ECD1 (Unit for time: sec)

\begin{tabular}{lcccccc}
\hline Capacity interval (ton/time) & 50 & 150 & 250 & 350 & 450 & 550 \\
\hline Num. of upper BD iterations & 113 & 97 & 93 & 71 & 65 & 69 \\
Num. of upper DWD iterations & 56 & 48 & 46 & 35 & 32 & 34 \\
Num. of lower DWD iterations & 209 & 186 & 179 & 138 & 131 & 130 \\
Num. of pricing problems solved \# & 265 & 234 & 225 & 173 & 163 & 163 \\
Optimal obj. (Billion \$) & -20.33 & -20.22 & -19.68 & -19.56 & -19.66 & -19.63 \\
Time for pricing problems & 2514 & 2218 & 1925 & 1543 & 1491 & 1324 \\
Time for DW restricted MP & 587 & 402 & 345 & 204 & 191 & 177 \\
Time for BD relaxed MP & 1.3 & 0.4 & 0.4 & 0.6 & 0.4 & 0.4 \\
Total solver time & 3103 & 2621 & 2270 & 1747 & 1683 & 1501 \\
Wall time & 5175 & 4255 & 3720 & 2862 & 2775 & 2458 \\
\hline
\end{tabular}

Table 14: Computational Results of Case C - ECD2 (Unit for time: sec)

\begin{tabular}{lcccccc}
\hline Capacity interval (ton/time) & 50 & 150 & 250 & 350 & 450 & 550 \\
\hline Num. of upper BD iterations & 61 & 54 & 51 & 40 & 37 & 39 \\
Num. of upper DWD iterations & 4 & 4 & 4 & 4 & 4 & 4 \\
Num. of lower DWD iterations & 154 & 146 & 134 & 102 & 103 & 99 \\
Num. of pricing problems solved \# & 158 & 150 & 138 & 106 & 107 & 103 \\
Optimal obj. (Billion \$) & -20.32 & -20.22 & -19.68 & -19.56 & -19.66 & -19.63 \\
Time for pricing problems & 1785 & 1699 & 1422 & 1106 & 1138 & 1148 \\
Time for DW restricted MP & 465 & 369 & 294 & 171 & 171 & 232 \\
Time for BD relaxed MP & 0.6 & 0.5 & 0.4 & 0.3 & 0.3 & 0.3 \\
Total solver time & 2250 & 2069 & 1716 & 1277 & 1308 & 1380 \\
Wall time & 3610 & 3356 & 2792 & 2028 & 2129 & 2149 \\
\hline
\end{tabular}

\#: Total number of IPP, DWPP, DWFP, DWPP-R and DWFP-R solved 

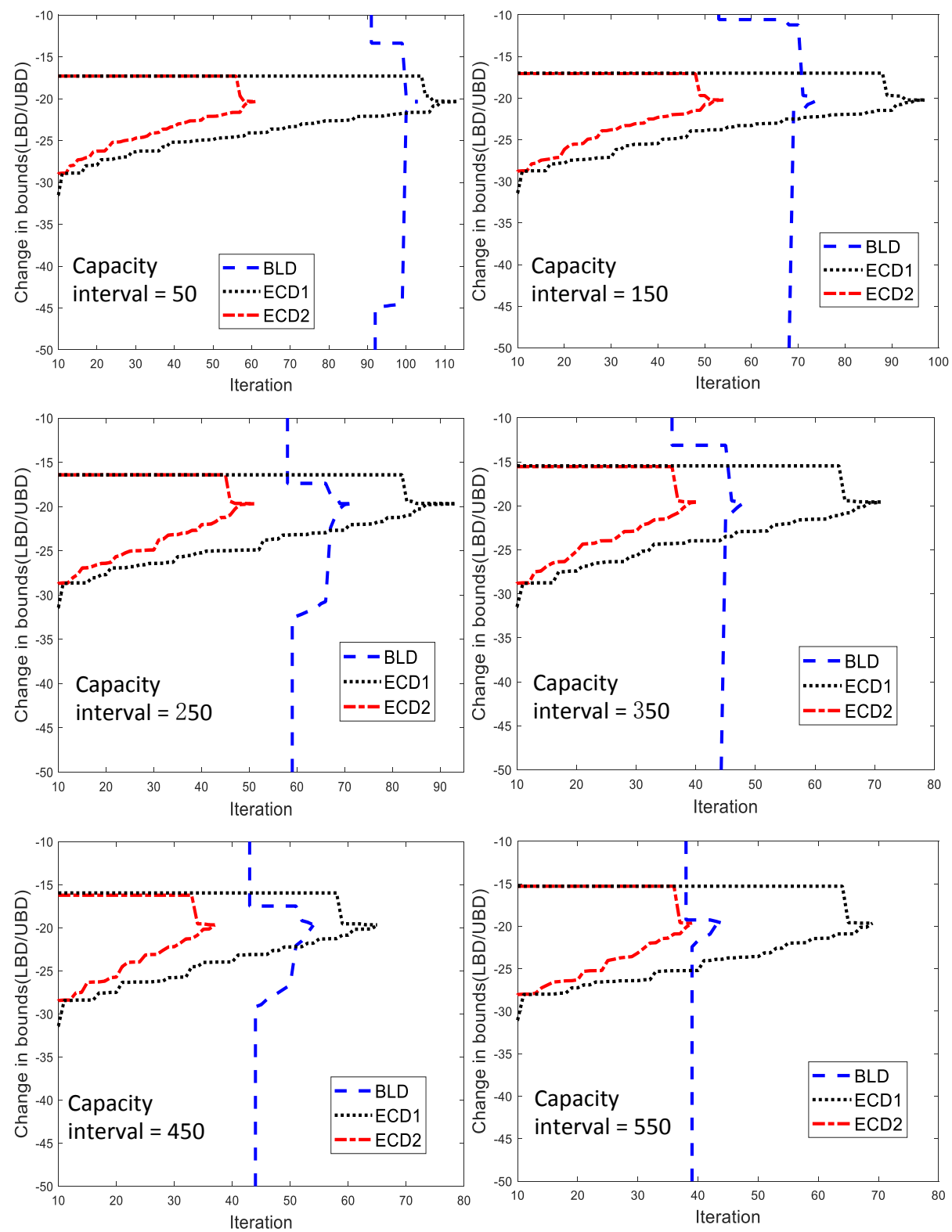

Figure 10: Comparison of bound evolution in different methods - Case C 


\section{Conclusions}

Exploitation of problem structure is often the key to efficient solution of problems that cannot be solved efficiently by off-the-shelf optimization solvers, but the classical decomposition methods can only deal with problems with weak linking constraints. In this paper, we develop two types of decomposition methods for MILPs with both strong and weak linking constraints. The first method (represented by BLD) follows a natural two level decomposition framework, which employs BD for the upper level decomposition and DWD for the lower level decomposition. The second method (represented by ECD) follows the same bilevel strategy, but uses CD (rather than BD) in the upper level decomposition. Both decomposition methods can terminate finitely with an $\epsilon$-optimal solution or a certificate of infeasibility. We further develop two variants of ECD (represented by ECD1 and ECD2), depending on how to alter between BD and DWD in the upper level decomposition. We also demonstrate how to apply BLD and ECD to risk-averse two-stage stochastic programing problems including CVaR constraints, which is a typical example of MILP with both strong and weak linking constraints.

The case study results show that the solution of the risk-averse two-stage stochastic programming formulation is more robust than that of the classical risk-neutral formulation. The results also demonstrate the computational advantages of the three proposed decomposition methods (i.e., BLD, ECD1, ECD2) over the monolith approach. Among the three decomposition methods, BLD tends to give the best performance when the problem needs only a small number of iterations to converge (such as some problem instances in Case C). This is because in this situation, using $\mathrm{CD}$ instead of $\mathrm{BD}$ in the upper level decomposition does not reduce the number of iteration significantly but requires solving more subproblems. However, when the problem is more difficult (either due to more continuous variables or more candidate integer decisions), using CD in the upper level is more advantageous and therefore ECD1 and ECD2 may outperform BLD (such as in Case A). In addition, ECD2 is almost always better than ECD1, because ECD2 is able to avoid ineffective CD iterations (i.e., the CD iterations that cannot reduce the gap) while ECD1 is not. 
492

493

494

495

496

\section{Acknowledgement}

The authors are grateful to the financial support from Natural Sciences and Engineering

Research Council of Canada through the Discovery Grant RGPIN 418411-13 and the Collaborative Research and Development Grant CRDPJ 485798-15. 


\section{List of Abbreviations}

BD

BLD

BMP

CD

$\mathrm{VaR}$

CVaR

DWD

DWMP

ECD

GUSS

LD

LP

MILP

RLD

$\mathrm{SCO}$

$\mathrm{SP}$

$\mathrm{BFP}^{k}$

$\mathrm{BPP}^{k}$

BRMP-BLD $^{k}$

${\mathrm{BRMP}-\mathrm{ECD}^{k}}^{k}$

DWRMP-ECD ${ }^{k}$

DWFRMP-ECD ${ }^{k}$

DWPP $^{k}$

DWFP $^{k}$

DWRMP-R $^{k, l}$

DWFRMP-R $^{k, l}$

DWPP-R ${ }^{k, l}$

DWFP-R $^{k, l}$

IPP
Benders decomposition

Bilevel decomposition

Benders master problem

Cross decomposition

Value-at-Risk

Conditional Value-at-Risk

Dantzig-Wolfe Decomposition

Dantzig-Wolfe Master Problem

Extended Cross Decomposition

Gather-Update-Solve-Scatter

Lagrangian Decomposition

Linear Programming

Mixed-integer Linear Program/Programming

Restricted Lagrangian Dual Problem

Supply Chain Optimization

Stochastic Programming

\section{Subproblems solved}

Benders feasibility problem at iteration $k$

Benders primal problem at iteration $k$

Benders relaxed master problem at iteration $k$ of BLD

Benders relaxed master problem at iteration $k$ of ECD

Dantzig-Wolfe restricted master problem at iteration $k$ of ECD

Dantzig-Wolfe feasibility restricted master problem at iteration $k$ of ECD

Dantzig-Wolfe pricing problem at iteration $k$

Dantzig-Wolfe feasibility pricing at iteration $k$

Dantzig-Wolfe restricted master problem at the $k t h$ upper level iteration and $l$ th lower level iteration

Dantzig-Wolfe feasibility restricted master problem at the $k t h$ upper level iteration and $l t h$ lower level iteration

Dantzig-Wolfe pricing problem at the $k t h$ upper level iteration and $l$ th lower level iteration

Dantzig-Wolfe feasibility pricing problem at the $k t h$ upper level iteration and $l$ th lower level iteration

Initial pricing problem 


\section{Appendix}

\section{A. ECD subproblems for CVaR constrained two-stage stochastic programming}

We show the ECD subproblems for solving Problem (CVaR-SP). In ECD, we treat $\left(x_{0}, \zeta\right)$ as linking variables. First, the following subproblem is solved to provide the initial point or column for the algorithm to commence.

$$
\begin{aligned}
\min _{x_{\omega}, \psi_{\omega}} & c_{\omega}^{\mathrm{T}} x_{\omega} \\
& x_{\omega} \in X_{\omega} .
\end{aligned}
$$

Let $x_{\omega}^{\star}$ be the optimal solution of Problem $\left(\mathrm{CVaR}^{-I P P}{ }_{\omega}\right)$. The initial column used to start the algorithm is $\left(x_{\omega}^{0}, \psi_{\omega}^{0}\right)=\left(x_{\omega}^{\star}, 0\right)$.

\section{A.1 Phase I subproblems}

The DW restricted master problem to solve at the upper level for Phase I is given as:

$$
\begin{aligned}
& \min _{\substack{x_{0}, \zeta \\
\theta^{0}, \ldots, \theta^{t-1} \geq 0 \\
z_{1,1,1}, \ldots, z_{2, s}, \ldots, z_{2, s} \\
z_{2,1}, z_{3}}}\left\|\left(z_{1,1}, \ldots, z_{1, s}\right)\right\|+\left\|\left(z_{2,1}, \ldots, z_{2, s}\right)\right\|+\left\|z_{3}\right\| \\
& \text { s.t. } A_{\omega} x_{0}+A_{\omega} \sum_{i=0}^{t-1} \theta^{i} x_{\omega}^{i} \leq b_{0, \omega}+z_{1, \omega}, \quad \omega \in\{1, \ldots, s\} \\
& -\sum_{i=0}^{t-1} \theta^{i} \psi_{\omega}{ }^{i}+f_{\omega}\left(\sum_{i=0}^{t-1} \theta^{i} x_{\omega}^{i}\right)-\zeta \leq z_{2, \omega}, \quad \omega \in\{1, \ldots, s\}, \quad\left(\text { CVaR-DWFRMP }^{k}\right) \\
& \zeta+\sum_{\omega=1}^{s} \hat{p}_{\omega}\left(\sum_{i=0}^{t-1} \theta^{i} \psi_{\omega}^{i}\right) \leq b_{0}+z_{3} \\
& \sum_{i=0}^{t-1} \theta^{i}=1 \text {, } \\
& x_{0} \in X_{0}, \zeta \in \mathbb{R}, z_{1, \omega}, z_{2, \omega} \geq 0, \forall \omega \in\{1, \ldots, s\}, z_{3} \geq 0 .
\end{aligned}
$$

Let $\mu_{1, \omega}^{k}, \mu_{2, \omega}^{k}$ be the Lagrange multiplier associated with the weak linking constraints (first and second group of constraints), and, $\mu_{3}^{k}$ be associated with the strong linking constraints (third group of constraints). 
The DW pricing problem for Phase I is given below:

$$
\begin{gathered}
\min _{x_{\omega}, \psi_{\omega}}\left(\mu_{1, \omega}^{k}\right)^{\mathrm{T}} A_{\omega} x_{\omega}+\mu_{2, \omega}^{k}\left(-\psi_{\omega}+f_{\omega}\left(x_{\omega}\right)\right)+\left(\mu_{3}^{k}\right) \hat{p}_{\omega} \psi_{\omega} \\
x_{\omega} \in X_{\omega}, \psi_{\omega} \geq 0 .
\end{gathered}
$$

$\left(\mathrm{CVaR}-\mathrm{DWFP}{ }_{\omega}^{k}\right)$

505 Let the solution be given by $\left(x_{\omega}^{k}, \psi_{\omega}^{k}\right)$ and the optimal objective $o b j_{C V a R-D W F P^{k}}=\sum_{\omega=1}^{s} o b j_{C V a R-D W F P_{\omega}^{k}}$.

506 The solution to Problem (CVaR-DWFP $\left.{ }_{\omega}^{k}\right)$ provides a column for Problem $\left(\right.$ CVaR-DWFRMP $\left.{ }^{k}\right)$.

The DW restricted master problem at the lower level can be written as:

$$
\begin{array}{ll}
\underset{\substack{\theta^{0}, \ldots, \theta^{l-1} \\
z_{1,1}, \ldots, z_{2, s} \\
z_{2,1}, \ldots, z_{2, s}}}{z_{3}} & \left\|\left(z_{1,1}, \ldots, z_{1, s}\right)\right\|+\left\|\left(z_{2,1}, \ldots, z_{2, s}\right)\right\|+\left\|z_{3}\right\| \\
\text { s.t. } & A_{\omega} \sum_{i=0}^{l-1} \theta^{i} x_{\omega}^{k, i} \leq b_{0, \omega}-A_{\omega} x_{0}^{k}+z_{1, \omega}, \quad \omega \in\{1, \ldots, s\}, \\
& -\sum_{i=0}^{l-1} \theta^{i} \psi_{\omega}^{k, i}+f_{\omega}\left(\sum_{i=0}^{l-1} \theta^{i} x_{\omega}^{k, i}\right) \leq \zeta^{k}+z_{2, \omega}, \quad \omega \in\{1, \ldots, s\}, \quad\left(\mathrm{CVaR}_{-} \mathrm{DWFRMPR}^{k, l}\right) \\
& \sum_{\omega=1}^{s} \hat{p}_{\omega}\left(\sum_{i=0}^{l-1} \theta^{i} \psi_{\omega}^{k, i}\right) \leq-\zeta^{k}+b_{0}+z_{3}, \\
& \sum_{i=0}^{l-1} \theta^{i}=1, \\
& z_{1, \omega}, z_{2, \omega} \geq 0, \forall \omega \in\{1, \ldots, s\}, z_{3} \geq 0 .
\end{array}
$$

${ }_{507}$ Let $\mu_{3}^{k, l}$ be the Lagrange multiplier associated with the strong linking constraints for $\left(\mathrm{CVaR}-\mathrm{DWFRMPR}{ }^{k, l}\right)$.

The DW pricing problem at the lower level can be written as:

$$
\begin{array}{ll}
\min _{x_{\omega}, \psi_{\omega}, z_{1, \omega}, z_{2, \omega}}\left(\mu_{3}^{k, l}\right) \hat{p}_{\omega} \psi_{\omega}+\left\|z_{1, \omega}\right\|+\left\|z_{2, \omega}\right\| \\
\text { s.t. } & A_{\omega} x_{\omega} \leq-A_{0, \omega} x_{0}^{k}+b_{0, \omega}+z_{1, \omega} \\
& -\psi+f_{\omega}\left(x_{\omega}\right) \leq \zeta^{k}+z_{2, \omega} \\
& x_{\omega} \in X_{\omega}, \psi_{\omega} \geq 0 \\
& z_{1, \omega}, z_{2, \omega} \geq 0
\end{array}
$$


Again, let the solution of $\left(\mathrm{CVaR}^{-D W F P}{ }_{\omega}^{k}\right)$ be given by $\left(x_{\omega}^{k, l}, \psi_{\omega}^{k, l}\right)$ and the optimal objective $o b j_{C V a R-D W F P R^{k, l}}=\sum_{\omega=1}^{s} o b j_{C V a R-D W F P R_{\omega}^{k, l}}$. The solution provides a column for Problem (CVaR-DWFRMPR $\left.{ }^{k, l}\right)$. An initial pricing problem is solved to generate a starting column for the DWD procedure and was given previously.

The relaxed master problem to solve at Phase I is now given as the following:

$$
\begin{array}{ll}
\min _{x_{0}, \zeta, \eta} \eta & \\
\text { s.t. } & \eta \geq o b j_{C V a R-D W F P}+\sum_{\omega=1}^{s}\left(\mu_{1, \omega}\right)^{\mathrm{T}} A_{0, \omega} x_{0}-\sum_{\omega=1}^{s}\left(\mu_{1, \omega}{ }^{i}\right) b_{0, \omega}-\sum_{\omega=1}^{s}\left(\mu_{2, \omega}{ }^{i}\right) \zeta \\
& +\mu_{3}^{i} \zeta-\mu_{3}^{i} b_{0}, \quad \forall i \in U_{\text {feas }}^{k}, \\
& \eta \geq o b j_{C V a R-D W F P R^{i, j}}+\sum_{\omega=1}^{s}\left(\mu_{1, \omega}{ }^{i, j}\right)^{\mathrm{T}} A_{0, \omega}\left(x_{0}-x_{0}^{i}\right)-\sum_{\omega=1}^{s}\left(\mu_{2, \omega}{ }^{i, j}\right)\left(\zeta-\zeta^{i}\right) \\
& +\left(\mu_{3}^{i, j}\right) \zeta-\left(\mu_{3}^{i, j}\right) b_{0}, \quad \forall(i, j) \in T_{\text {feas }}^{k}, \\
& x_{0} \in X_{0}, \zeta \in \mathbb{R}, \eta \in \mathbb{R},
\end{array}
$$

$\left(\right.$ CVaR-BRMPI $\left.^{k}\right)$

\section{A.2 Phase II subproblems}

In Phase II, the DW restricted master problem solved at the upper level is:

$$
\begin{array}{ll} 
& \min _{\substack{x_{0}, \zeta \\
\theta^{0}, \ldots, \theta^{t-1} \geq 0}} \sum_{\omega=1}^{s}\left(c_{0, \omega}^{\mathrm{T}} x_{0}+c_{\omega}^{\mathrm{T}}\left(\sum_{i=0}^{t-1} \theta^{i} x_{\omega}^{i}\right)\right) \\
\text { s.t. } & A_{\omega} x_{0}+A_{\omega} \sum_{i=0}^{t-1} \theta^{i} x_{\omega}^{i} \leq b_{0, \omega}, \quad \omega \in\{1, \ldots, s\}, \\
& -\sum_{i=0}^{t-1} \theta^{i} \psi_{\omega}^{i}+f_{\omega}\left(\sum_{i=0}^{t-1} \theta^{i} x_{\omega}^{i}\right)-\zeta \leq 0, \quad \omega \in\{1, \ldots, s\}, \quad\left(\mathrm{CVaR}^{i} \mathrm{DWRMP}^{k}\right) \\
& \zeta+\sum_{\omega=1}^{s} \hat{p}_{\omega}\left(\sum_{i=0}^{t-1} \theta^{i} \psi_{\omega}^{i}\right) \leq b_{0}, \\
& \sum_{i=0}^{t-1} \theta^{i}=1, \\
& x_{0} \in X_{0}, \zeta \in \mathbb{R} .
\end{array}
$$


Let $\lambda_{1, \omega}^{k}, \lambda_{2, \omega}^{k}$, and $\lambda_{3}^{k}$, be the Lagrange multiplier associated with the first, second, and third group of constraints of $\left(\mathrm{CVaR}-\mathrm{DWRMP}{ }^{k}\right)$. The pricing problem is the following decomposable problem:

$$
\begin{gathered}
\min _{x_{\omega}, \psi_{\omega}}\left(c_{\omega}^{\mathrm{T}}+\left(\lambda_{1, \omega}^{k}\right)^{\mathrm{T}} A_{\omega}\right) x_{\omega}+\lambda_{2, \omega}^{k}\left(-\psi_{\omega}+f_{\omega}\left(x_{\omega}\right)\right)+\left(\lambda_{3}^{k}\right) \hat{p}_{\omega} \psi_{\omega} \\
x_{\omega} \in X_{\omega}, \psi_{\omega} \geq 0 .
\end{gathered}
$$

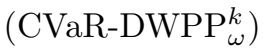

${ }_{513}$ Let the solution of $\left(\mathrm{CVaR}-\mathrm{DWPP}{ }_{\omega}^{k}\right)$ be given by $\left(x_{\omega}^{k}, \psi_{\omega}^{k}\right)$ and the optimal objective ${ }_{514} o b j_{C V a R-D W P P^{k}}=\sum_{\omega=1}^{s} o b j_{C V a R-D W P P_{\omega}^{k}}$. The solution provides a column for Problem $\left(\mathrm{CVaR}-\mathrm{DWRMP}{ }^{k}\right)$.

The Phase II DW restricted master problem solved at the lower level is given as:

$$
\begin{array}{ll} 
& \min _{\theta^{0}, \ldots, \theta^{l-1} \geq 0} \sum_{\omega=1}^{s}\left(c_{0, \omega}^{\mathrm{T}} x_{0}^{k}+c_{\omega}^{\mathrm{T}}\left(\sum_{i=0}^{l-1} \theta^{i} x_{\omega}^{k, i}\right)\right) \\
\text { s.t. } & A_{\omega} \sum_{i=0}^{l-1} \theta^{i} x_{\omega}^{k, i} \leq b_{0, \omega}-A_{\omega} x_{0}^{k}, \quad \omega \in\{1, \ldots, s\}, \\
& -\sum_{i=0}^{l-1} \theta^{i} \psi_{\omega}^{k, i}+f_{\omega}\left(\sum_{i=0}^{l-1} \theta^{i} x_{\omega}^{k, i}\right) \leq \zeta^{k}, \quad \omega \in\{1, \ldots, s\}, \quad\left(\mathrm{CVaR}^{k} \mathrm{DWRMPR}{ }^{k, l}\right) \\
& \sum_{\omega=1}^{s} \hat{p}_{\omega}\left(\sum_{i=0}^{l-1} \theta^{i} \psi_{\omega}^{k, i}\right) \leq-\zeta^{k}+b_{0}, \\
& \sum_{i=0}^{l-1} \theta^{i}=1,
\end{array}
$$

${ }_{516}$ Let $\lambda_{3}^{k, l}$ be the Lagrange multiplier associated with the strong linking constraints. Sim517 ilarly, the Phase II DW pricing problem solved at the lower level is the following:

$$
\begin{array}{ll}
\min _{x_{\omega}, \psi_{\omega}} & \left(c_{\omega}^{\mathrm{T}}+\left(\lambda_{1, \omega}^{k}\right)^{\mathrm{T}} A_{\omega}\right) x_{\omega}+\lambda_{3}^{k}\left(\hat{p}_{\omega} \psi_{\omega}\right) \\
\text { s.t. } & A_{\omega} x_{\omega}=-A_{0, \omega} x_{0}^{k}+b_{0, \omega}, \quad \omega \in\{1, \ldots, s\}, \\
& -\psi_{\omega}+f_{\omega}\left(x_{\omega}\right) \leq \zeta^{k}, \quad \omega \in\{1, \ldots, s\}, \\
& x_{\omega} \in x_{\omega}, \psi_{\omega} \geq 0 .
\end{array}
$$


518

519

Again let the solution of Problem $\left(\mathrm{CVaR}-\mathrm{DWPPR} \mathrm{R}_{\omega}^{k, l}\right)$ be given by $\left(x_{\omega}^{k, l}, \psi_{\omega}^{k, l}\right)$ with the optimal objective $o b j_{C V a R-D W P P R^{k, l}}=\sum_{\omega=1}^{s} o b j_{C V a R-D W P P R_{\omega}^{k, l}}$. The solution provides a column for Problem $\left(\mathrm{CVaR}-\mathrm{DWRMPR}{ }^{k, l}\right)$. The Phase II relaxed master problem, a lower bound to Problem (CVaR-SP) is the following:

$$
\begin{aligned}
& \min _{x_{0}, \zeta, \eta} \eta \\
& \text { s.t. } \quad \eta \geq o b j_{C V a R-D W P P^{i}}+\sum_{\omega=1}^{s}\left(c_{0, \omega}^{\mathrm{T}}+\left(\lambda_{1, \omega}{ }^{i}\right)^{\mathrm{T}} A_{0, \omega}\right) x_{0}-\sum_{\omega=1}^{s}\left(\lambda_{1, \omega}{ }^{i}\right)^{\mathrm{T}} b_{0, \omega} \\
& -\sum_{\omega=1}^{s} \lambda_{2, \omega}{ }^{i} \zeta+\lambda_{3}^{i}\left(\zeta-b_{0}\right), \quad \forall i \in U_{o p t}^{k} \\
& 0 \geq o b j_{C V a R-D W F P^{i}}+\sum_{\omega=1}^{s}\left(\mu_{1, \omega}{ }^{i}\right)^{\mathrm{T}} A_{0, \omega} x_{0}-\sum_{\omega=1}^{s}\left(\mu_{1, \omega}{ }^{i}\right)^{\mathrm{T}} b_{0, \omega}-\sum_{\omega=1}^{s} \mu_{2, \omega}{ }^{i} \zeta \\
& +\mu_{3}^{i}\left(\zeta-b_{0}\right), \forall i \in U_{\text {feas }}^{k}, \\
& \eta \geq o b j_{C V a R-D W P P R^{i, j}}+\sum_{\omega=1}^{s} c_{0, \omega}^{\mathrm{T}} x_{0}+\sum_{\omega=1}^{s}\left(\lambda_{1, \omega}{ }^{i, j}\right)^{\mathrm{T}} A_{0, \omega}\left(x_{0}-x_{0}^{i}\right) \\
& -\sum_{\omega=1}^{s} \lambda_{2, \omega}{ }^{i, j}\left(\zeta-\zeta^{i}\right)+\lambda_{3}^{i, j}\left(\zeta-b_{0}\right), \quad \forall(i, j) \in T_{o p t}^{k} \\
& 0 \geq o b j_{C V a R-D W F P R}{ }^{i, j}+\sum_{\omega=1}^{s}\left(\mu_{1, \omega}{ }^{i, j}\right)^{\mathrm{T}} A_{0, \omega}\left(x_{0}-x_{0}^{i}\right)-\sum_{\omega=1}^{s} \mu_{2, \omega}{ }^{i, j}\left(\zeta-\zeta^{i}\right) \\
& +\mu_{3}^{i, j}\left(\zeta-b_{0}\right), \forall(i, j) \in T_{\text {feas }}^{k}, \\
& x_{0} \in X_{0}, \zeta \in \mathbb{R}, \eta \in \mathbb{R} .
\end{aligned}
$$

$\left(\right.$ CVaR-BRMPII $\left.^{k}\right)$ 
B. BLD subproblems for CVaR constrained two-stage stochastic programming

The lower level subproblems for the bilevel decomposition are same to those for ECD. At the upper level, however, the following Benders relaxed master problem is solved:

$$
\begin{array}{ll}
\min _{x_{0}, \zeta, \eta} \eta & \\
\text { s.t. } \quad & \eta \geq o b j_{C V a R-D W P P R^{i, j}}+\sum_{\omega=1}^{s} c_{0, \omega}^{\mathrm{T}} x_{0}+\sum_{\omega=1}^{s}\left(\lambda_{1, \omega}{ }^{i, j}\right)^{\mathrm{T}} A_{0, \omega}\left(x_{0}-x_{0}^{i}\right) \\
& -\sum_{\omega=1}^{s} \lambda_{2, \omega}{ }^{i, j}\left(\zeta-\zeta^{i}\right)+\lambda_{3}^{i, j}\left(\zeta-b_{0}\right), \quad \forall(i, j) \in T_{o p t}^{k}, \\
& 0 \geq o b j_{C V a R-D W F P R^{i, j}}+\sum_{\omega=1}^{s}\left(\mu_{1, \omega}{ }^{i, j}\right)^{\mathrm{T}} A_{0, \omega}\left(x_{0}-x_{0}^{i}\right)-\sum_{\omega=1}^{s} \mu_{2, \omega}{ }^{i, j}\left(\zeta-\zeta^{i}\right) \\
& +\mu_{3}^{i, j}\left(\zeta-b_{0}\right), \forall(i, j) \in T_{\text {feas }}^{k}, \\
& x_{0} \in X_{0}, \zeta \in \mathbb{R}, \eta \in \mathbb{R} .
\end{array}
$$

$\left(\right.$ CVaR-BRMP-BLD $\left.{ }^{k}\right)$

\section{References}

[1] F. You, I. E. Grossmann, Multicut Benders decomposition algorithm for process supply chain planning under uncertainty, Annals of Operations Research 210 (1) (2013) 191211.

[2] X. Li, E. Armagan, A. Tomasgard, P. I. Barton, Stochastic pooling problem for natural gas production network design and operation under uncertainty, AIChE Journal 57 (2011) 2120-2135.

[3] W. Li, C.-W. Hui, P. Li, A.-X. Li, Refinery planning under uncertainty, Industrial \& Engineering Chemistry Research 43 (21) (2004) 6742-6755.

[4] N. Sahinidis, I. Grossmann, Convergence properties of generalized Benders decomposition, Computers and Chemical Engineering 15 (7) (1991) 481 - 491.

[5] J. R. Birge, F. Louveaux, Introduction to stochastic programming, Springer series in operations research, Springer, New York, 1997. 
[6] N. Miller, A. Ruszczynski, Risk-averse two-stage stochastic linear programming: Modeling and decomposition, Operations Research 59 (2011) 125-132.

[7] N. Noyan, Risk-averse two-stage stochastic programming with an application to disaster management, Computers and Operations Research 39 (2012) 541-559.

[8] S. Ahmed, M. Tawarmalani, N. V. Sahinidis, A finite branch-and-bound algorithm for two-stage stochastic integer programs, Mathematical Programming 100 (2) (2004) $355-377$.

[9] A. Barbaro, M. J. Bagajewicz, Managing financial risk in planning under uncertainty, AIChE Journal 50 (5) (2004) 963-989.

[10] R. Rockafellar, S. Uryasev, Optimization of conditional value-at-risk, Journal of Risk 2 (2000) 21-41.

[11] X. Liu, S. Küçükyavuz, J. Luedtke, Decomposition algorithms for two-stage chanceconstrained programs, Mathematical Programming 157 (1) (2016) 219-243.

[12] Q. Wang, Y. Guan, J. Wang, A chance-constrained two-stage stochastic program for unit commitment with uncertain wind power output, IEEE Transactions on Power Systems 27 (1) (2012) 206-215.

[13] A. Charnes, W. W. Cooper, Chance-constrained programming, Management Science 6 (1) (1959) 73-79.

[14] J. Benders, Partitioning procedures for solving mixed-variables programming problems, Numerische Mathematik 4 (1962) 238-252.

[15] R. M. V. Slyke, R. Wets, L-shaped linear programs with applications to optimal control and stochastic programming, SIAM Journal on Applied Mathematics 17 (1969) 638663.

[16] G. Dantzig, P. Wolfe, The decomposition principle for linear programs, Operations Research 8 (1960) 101-111. 
[17] L. H. Applegren, A column generation algorithm for a ship scheduling problem, Transportation Science 3 (1969) 53-68.

[18] A. M. Geoffrion, Lagrangean relaxation for integer programming, Mathematical Programming Study 2 (1974) 82-114.

[19] M. Fisher, Lagrangian relaxation methods for solving integer programming problems, Management Science 27 (1981) 1-18.

[20] S. Bruno, C. Sagastizábal, Optimization of real asset portfolio using a coherent risk measure:Application to oil and energy industries, Optim. Eng. 12 (2011) 257-275.

[21] E. Ogbe, X. Li, A new cross decomposition method for stochastic mixed-integer linear programming, European Journal of Operational Research 256 (2) (2017) 487 - 499.

[22] T. J. Van Roy, Cross decomposition for mixed integer programming, Mathematical programming 25 (1) (1983) 46-63.

[23] K. Holmberg, On the convergence of cross decomposition, Mathematical Programming 47 (1990) $269-296$.

[24] S. Mitra, P. Garcia-Herreros, I. E. Grossmann, A cross-decomposition scheme with integrated primal-dual multi-cuts for two-stage stochastic programming investment planning problems, Mathematical Programming 157 (1) (2016) 95-119.

[25] S. R. Cardoso, A. P. Barbosa-Póvoa, S. Relvas, Integrating financial risk measures into the design and planning of closed-loop supply chain, Computers and Chemical Engineering 85 (2016) $105-123$.

[26] P. Krokhmal, J. Palmquist, S. Uryasev, Portfolio optimization with conditional valueat-risk objective and constraints, Journal of Risk 4 (2002) 11-27.

[27] R. Rockafellar, S. Uryasev, Conditional value-at-risk for general loss distribution, Journal of Banking and Finance 26 (2002) 1443-1471. 
[28] Z. Huang, Q. P. Zheng, Decomposition-based exact algorithms for risk-constrained traveling salesman problems with discrete random arc costs, Optimization Letters 9 (8) (2015) 1553-1568.

[29] S. Ahmed, Convexity and decomposition of mean-risk stochastic programs, Math. Program. 106 (2006) 433-446.

[30] R. Schultz, S. Tiedemann, Conditional value-at-risk in stochastic programs with mixedinteger recourse, Mathematical Programming 105 (2006) 365-386.

[31] Q. Zhang, J. L. Cremer, I. E. Grossmann, A. Sundaramoorthy, J. M. Pinto, Risk-based integrated production scheduling and electricity procurement for continuous powerintensive processes, Computers and Chemical Engineering 86 (2016) 90 - 105.

[32] M. Carneiro, G. Ribas, , S. Hamacher, Risk management in oil and gas industries: A CVaR approach, Industrial \& Engineering Chemistry Research 49 (2010) 3286-3294.

[33] P. Verderame, C. Floudas, Multisite planning under demand and transportation time uncertainty: Robust optimization and conditional value-at-risk frameworks, Industrial and Engineering Chemistry Research 50 (2010) 4959-4982.

[34] P. Verderame, C. Floudas, Operational planning of large-scale industrial batch plants under demand and amount uncertainty: II. conditional value-at-risk framework, Industrial and Engineering Chemistry Research 49 (2010) 260-275.

[35] C. Fabian, A. Veszpremi, Algorithms for handling CVaR constraints in dynamic stochastic programming models with applications to finance, Journal of Risk 10 (2008) $111-131$.

[36] A. Brook, D. Kendrick, A. Meeraus, GAMS, a user's guide, SIGNUM Newsletter 23 (34) (1988) 10-11.

[37] IBM, IBM ILOG CPLEX OPTIMIZER: High-performance mathematical programming engine. (2014). 
[38] M. R. Bussieck, M. C. Ferris, T. Lohmann, GUSS: Solving collections of data related models within GAMS, in: J. Kallrath (Ed.), Algebraic Modeling Systems: Modeling and Solving Real World Optimization Problems, Springer Berlin Heidelberg, Berlin, Heidelberg, 2012, Ch. 3, pp. 35-56.

[39] L. Cucek, H. Lam, J. Klemes, P. Varbanov, Z. Kravanja, Synthesis of regional networks for the supply of energy and bioproducts, Clean. Technol. Environ. Policy 12 (2010) 635-645.

[40] K. McLean, X. Li, Robust scenario formulations for strategic supply chain optimization under uncertainty, Industrial and Engineering Chemistry Research 52 (2013) 57215734 .

[41] K. McLean, E. Ogbe, X. Li, Novel formulation and efficient solution strategy for strategic optimization of an industrial chemical supply chain under demand uncertainty, The Canadian Journal of Chemical Engineering 93 (6) (2015) 971-985. 